\title{
RECHERCHES SUR LA MÉTHODE DE GRAEFFE ET LES ZÉROS DES POLYNOMES ET DES SÉRIES DE LAURENT.
}

\author{
PAR \\ ALEXANDRE OSTROWSKI
}

à BÂLE.

Table des matières.

Bibliographie . . . . . . . . . . . . . . . . . . . . . . . 100

Introduction . . . . . . . . . . . . . . . . . . . . . . . . . . . 102

CHAPITRE I: Le diagramme de Newton et les séries normales.

§ I. Les polynômes normaux. (Nos. I- 5 , théorèmes I, II) . . . . . . 115

$\S$ 2. Le diagramme d'une série de Laurent. (Nos. 6-9, théorèmes III, IV) 118

$\S 3$. La majorante newtonienne, les inclinaisons numériques et les déviations.

(Nos. I o-I 2, théorème V) . . . . . . . . . . . . . . . . . . 120

$\S$ 4. Transformations du diagramme et relations caractéristiques. (Nos. I 3 - I 6 , théorème VI) . . . . . . . . . . . . . . . . . . . . 124

CHAPITRE II: Les bornes des racines dépendant des inclinaisons nuniériques du diagramme de Newton.

\$ 5. Premier théorème fondamental. (Nos. $x_{7}-23$, théorèmes VII, VIII) 132

$\S 6$. Corollaires du théorème fondamental. Equations des degrés 2 et 3 . (Nos. 24-28, théorèmes IX-XII) . . . . . . . . . . . . . . . 143

$\S$ 7. Evaluation des produits des racines. (Nos. 29-3r, théorèmes XIII-XV) 150

CHAPITRE III: Approximation et évaluation des racines dépendant des déviations.

$\S 8$. Séparation des racines pour les côtés simples du diagramme (deuxième théorème fondamental). (Nos. $32-38$, théorème XVI) . . . . . . 157

$\S 9$. Séparation simultanée de l'ensemble des racines. (Nos. $39^{--} 4 \mathrm{I}$, théorèmes

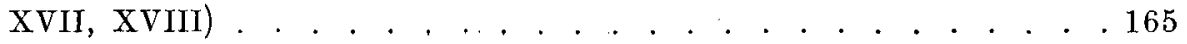


$\S$ Io. Séparation des groupes de racines en fonctions des déviations. (Nos. $42-45$, théorèmes $\mathrm{XIX}-\mathrm{XXI}$ ) . . . . . . . . . . . . . . . 171

$\S$ I I. Une évaluation du produit des racines pour $M>9$. (Nos. 46,47 , théorèmes XXII, XXIII) . . . . . . . . . . . . . . . . . . . 177

§ I 2. Le diagramme des produits de polynômes et de séries de Laurent. (Nos. 48-5 I, théorème XXIV) . . . . . . . . . . . . . . . . 181

§ I3. La factorisation de polynômes dans le cas de grandes déviations. (Nos. 52-56, thëorème XXV) . . . . . . . . . . . . . . . . 185

§ I 4. La factorisation des séries' de Laurent dans le cas de grandes déviations (troisième théorème fondamental). (Nos. 57-62, théorèmes XXVI, $\mathrm{XXVII})$. . . . . . . . . . . . . . . . . . . . . . 192

$\S$ I 5. Compléments au troisième théorème fondamental. (Nos. 63-68, théorèmes XXVIII, XXIX) . . . . . . . . . . . . . . . . . 200

CHAPITRE IV: Continuité des racines des équations algébriques. Méthode de Graeffe.

§ 6. Continuité des racines des équations algébriques (quatrième théorème fondamental). (Nos. 69-77, théorèmes XXX-XXXIII) . . . . 209

$\S$ I 7. La méthode de Graeffe. Calcul des modules des racines. (Nos. $78-86)$. . . . . . . . . . . . . . . . . . . 225

$\S$ I8. La méthode de Graeffe. Détermination complète des racines et exemples. (Nos. $87-95)$. . . . . . . . . . . . . . . 239

\section{Bibliographie.}

E. Carvallo: (I) Méthode pratique pour la résolution numérique complète des équations algébriques ou transcendantes. - Thèse de Paris I $890, \mathrm{pp}$. I-40.

G. Dandelin: ( $\mathrm{r}$ ) Recherches sur la résolution des équations numériques. - Nouveaux mémoires de l'académie royale des sciences et belles-lettres de Bruxelles, T. 3, I 826, pp. I-V et $\mathrm{I}-7 \mathrm{I}$; en particulier second supplément, pp. $45-6 \mathrm{I}$.

G. Dumas: ( $\mathrm{r}$ ) Sur les fonctions à caractère algébrique dans le voisinage d'un point donné. - Thèse de Paris I 904.

(2) Sur quelques cas d'irréductibilité des polynômes à coefficients rationnels. -Journal de mathématiques (6) 2 (1906), pp. i 9 I -258 .

J. F. Encke: (I) Allgemeine Auflösung der numerischen Gleichungen. - Berliner Astronomisches Jahrbuch 1841 , reproduit avec quelques additions dans le Journal de Crelle i841, Vol. 22, pp. 193-248. - La rédaction originale se 
trouve reproduite dans les oeuvres complètes d'Encke, Vol. I, Berlin I888, p. I 25-I87, et nos citations se rapportent à ce dernier endroit.

C. H. GraefFe: (I) Die Auflösung der höheren numerischen Gleichungen, als Beantwortung einer von der königlichen Akademie der Wissenschaften zu Berlin aufgestellten Preisfrage. - Zurich I 837 (Friedrich Schulthess), pp. I, II et I-44.

E. J. GumbeI: (I) Eine Darstellung statistischer Reihen durch Euler. - Jahreśbericht der Deutschen Mathematikervereinigung T. 25 (1917), pp. 25 $1-264$, en particulier pp. $259-262$.

J. Hadamard: (I) Étude sur les propriétés des fonctions entières et en particulier d'une fonction considérée par Riemann. - Journ. d. math. (4) I893, pp. I 7 I-2 r 5. - Réimprimé dans: J. Hadamard, Selecta, Paris, x 935, pp. 52-93.

- - (2) Sur les fonctions entières. - C. R. I35 (I902), pp. r309-13 Ir.

A. N. KrylofF: (I) Leçons sur les calculs approximatifs. 3-ième édition I 935 , Moscou (en russe), pp. I-54 I, en particulier pp. I $8-68$.

E. Landau: (I) Sur quelques théorèmes de M. Petrovitch relatifs aux zéros des fonctions analytiques. - Bull. soc. math. d. France, T. 33 (r905), pp. r-- I r.

-_-: (2) Ueber eine Aufgabe aus der Funktionentheorie. - Tôhoku math. Journ. T. 5 (I9I4), pp. 97-II6.

N. I. LobatschewskiJ: (I) Algebra. - Kasan 1834.

W. F. Osgood: (I) Lehrbuch der Funktionentheorie. - 'T. $\mathrm{II}_{1}$, Leipzig I 929.

A. Osтrowski: (r) Ueber algebraische Funktionen von Dirichletschen Reihen. Math. Zeitschrift T. 37 ( 9.33 ), pp. $98-\mathrm{r} 33$.

-- : (2) Sur les modules des zéros des fonctions entières. - C. R. 206 (1938), pp. I 54 I - I 543 .

- - : (3) Sur la continuité relative des racines d'équations algébriques. - C. R. 208 (1939), pp. $777^{-} 779$.

G. Polya: (I) Sur la méthode de Graeffe. - C. R. I56 (I9I3), pp. II45-i I 47 .

- - (2) Ueber das Graeffesche Verfahren. - Zeitschr. f. Math. u. Phys., T. 63 (1915), pp. 275-290.

C. Runge: (1) Praxis der Gleichungen. -- Seconde édition, Berlin I92 I.

C. RUNGe u. H. König: (I) Vorlesungen über numerisches Rechnen. - Berlin 1924.

G. Valiron: ( $\mathrm{I}$ ) Sur les fonctions entières d'ordre nul et d'ordre fini et en particulier les fonctions à correspondance régulière. - Thèse de Paris, I9I4, pp. I-I 4 I. - Réimprimé dans les Annales de la faculté des sciences de Toulouse (3) 5 , pp. I $7-259$. 


\section{Introduction.}

Dans le présent mémoire nous nous occupons particulièrement des propriétés des racines des polynômes et plus généralement des séries de Laurent, qui dépeudent en première ligne des modules des coefficients.

Les théorèmes généraux auquels nous arrivons dans ce mémoire sont appliqués dans les deux derniers $\S \S$ du mémoire à la méthode de Graeffe. C'est l'état assez rudimentaire dans lequel la théorie de cette méthode était restée jusqu'à aujourd'hui qui a provoqué ces recherches et a déterminé dans un certain degré les points de vue dont nous nous sommes laissés guider.

L'idée fondamentale de la méthode de résolution numérique des équations algébriques qu'on appelle aujourd'hui (avec raison) la méthode de Graeffe; a été formulée I 826 très clairement et tout à fait explicitement par Dandelin dans le second supplément à son mémoire fondamental sur les équations algébriques ${ }^{1}$, et quelques années plus tard, I834, par Lobatschewski².

Mais ce n'était que Graeffe ${ }^{3}$ qui, I 837, l'a mise au point et en a fait un procédé pratique, en ne poursuivant, il est vrai, qu'un seul des points de vue développés par Dandelin à l'endroit cité et en laissant complètement de côté un autre point de vue - celui de »décomposition» -, sur lequel Dandelin arait le plus insisté dans son mémoire.

Dans cette forme la méthode de Graeffe a été surtout popularisée grace à un mémoire d'Encke, le renom du célèbre astronome Berlinois ayant beaucoup contribué à faire cette méthode connue ${ }^{4}$.

1 DANDELIN (r) pp. 45-6r.

${ }^{2}$ LoBatsonewsKi (I).

${ }^{3}$ Graefre (1). Bien que le titre du mémoire de Graffre indique que ce mémoire est la solution d'un problème posé par l'académie de Berlin en 1836 pour le 3I. III. I838, l'académie était hors d'état de prendre en considération ce mémoire, puisqu'il était publié avant le terme, contrairement aux statuts, et l'académie n'avait reçu (en décembre 1836) de l'auteur qu'un exemplaire imprimé. Comme le seule mémoire jugé plus ou moins favorablement par l'académie traitait la méthode de GRA EFFE sans y apporter de progrès considérables, le terme était finalement prolongé jusqu'au 3r. IIl. 1839 et puis retiré définitivement, la moitié du prix de 50 ducates étant accordé à GraffFe, bien qu'il était considéré comme hors de coneours. (Cf. Abhandlungen der kgl. Akademie der Wissenschaften in Berlin I 838 pp. II-IV; I839 pp. VII-VIII).

"ENCKE (I). Toutefois dans son exposé ENCKE reclame "d'avoir complété la théorie par ce qui lui avait encore manqué pour la solution complète du problème». A savoir: ... mit der Ermittelung der imaginären Wurzeln selbst auf einfachem und strengem Wege, mit einer Erleichterung 
En I 890 E. Carvallo essaye dans sa thèse ${ }^{1}$ d'approfondir les bases théoriques de la méthode en reprenant le point de vue original de Dandelin; mais il parait que même le théorème de convergence servant de base à la méthode de Graeffe n'a été formulé nettement que par Pólya I9I 3 et $1915^{2}$.

Partons d'un polynôme

$$
f(z)=f_{0}(z) \equiv \prod_{v=1}^{n}\left(z-\zeta_{v}\right), \quad 0<\left|\zeta_{1}\right| \leqq\left|\zeta_{2}\right| \leqq \cdots \leqq\left|\zeta_{n}\right|
$$

et formons pour chaque entier $k \geqq 0$ la k-ième transformée de Graeffe de $f(z)$ :

$$
f_{k}(z) \equiv \prod_{\nu=1}^{n}\left(z-\zeta_{\nu}^{-k^{k}}\right)=z^{n}-a_{n-1}^{(k)} z^{n-1}+a_{n-2}^{(k)} z^{n-2}+\cdots
$$

Alors, si l'on a pour un $m:\left|\zeta_{m}\right|<\left|\zeta_{m+1}\right|$, on a avec $k \rightarrow \infty$ :

$$
\frac{a_{m}^{(k)}}{\left(\zeta_{m+1} \ldots \zeta_{n}\right)^{k}} \rightarrow \mathrm{I}^{3}
$$

La convergence étant très rapide, cette relation permet de calculer les modules de toutes les $\zeta_{v}$ en supposant que l'on connait tous les indices $m$ en question, ce qui n'est pas généralement le cas. L'idée ingénieuse et simple qui a permis à Graeffe de tourner cette difficulté consiste en ceci: On fait le calcul avec un certain nombre fixe de chiffres significatifs jusqu'à ce que dans le passage d'une transformée $f_{k}(z)$ à la transformée suivante les coefficients $a_{m}$ de certaines puissances $z^{m}$ de $z$ sont remplacés chaque fois par leur carrés. Alors il y a lieu de supposer que pour ces indices $m$ on a $\left|\zeta_{m}\right|<\left|\zeta_{m+1}\right|$. Mais en vérité tout ce qu'on puisse dire dans ce cas, c'est que pour les coefficients en

des Verfahrens bei Wurzeln, die nahe zusammenliegen und auch bei sehr hohen Potenzen sich nicht entscheidend genug trennen würden, und mit den Methoden die Werthe soweit der Wahrheit näher zu bringen, als man immer wünschen mag."

A notre avis ces reclamations sont extrèmement exagérées. Loin d'avoir ajouté quelques idées nouvelles au procédé de GRAEFFE, ENCKE a même omis quelques idées très utiles qui n'ont été repris que par Runge un demi siècle plus tard. Si l'exposé d'EnckE est parfois plus détaillé, il manque généralement de clarté et d'élégance de celui de GrakfFe. Les additions qui sont personnelles à ENcke ne se rapportent en vérité qu'aux points d'importance purement pratique, comme l'emploi des logarithmes etc.

1 Carvallo (i).

2 Pólya (1), (2).

- La relation correspondante à (3) est déduite dans Pólya (2) pour le cas le plus général d'une série entière, et on $\mathrm{y}$ trouve deux autres théorèmes de convergence apparentés à (3). Quant à la méthode de GRAEFFE pour le cas des séries entières on la trouve déjà appliquée aux exemples numériques dans Carvalio (I). 
question les ressources de calcul sont épuisées. Et puis, ce qui est encore plus grave, on utilise dans cette règle implicitement l'hypothèse que dans le calcul fait avec un certain nombre de chiffres, les erreurs "d'arrondissement» ne s'accumulent pas, ce qui est loin d'être vrai dans le cas le plus général. -

Les recherches exposées dans le présent mémoire attaquent le problème sous les 3 points de vue suivants:

A.) Il s'agit de déduire les valeurs des modules des racines de $f(z)$ en étudiant la suite des transformées de Graeffe $f_{k}(z)$.

B.) Il s'agit d'obtenir les valeurs complètes des racines en étudiant les $f_{k}(z)$.

C.) Le calcul exact des transformées $f_{k}(z)$ devenant très tôt impraticable, il s'agit d'étudier l'influence des serreurs d'arrondissement" sur les valeurs approchées des racines.

Examinons d'abord le problème A.). Il se décompose en trois problèmes partiels suivants:

$A \propto$.) Déduire les modules des racines avec une approximation donnée d'avance de la suite des $f_{k}(z)$ sans connaitre les indices $m$ avec $\left|\zeta_{m}\right|<\left|\zeta_{m+1}\right|$.

Aß.) Déterminer les indices $m$ avec $\left|\zeta_{m}\right|<\left|\zeta_{m+1}\right|$.

A $\gamma$.) Pour les indices $m$ en question trouver une évaluation de la différence

$$
\sqrt[2^{k}]{\left|a_{m}^{(k)}\right|}-\left|\zeta_{m+1} \ldots \zeta_{n}\right|
$$

Pour attaquer le problème $\mathbf{A} \alpha$.) nous nous posons le problème suivant: Supposons que dans l'équation algébrique

$$
F(z) \equiv A_{n} z^{n}+A_{n-1} z^{n-1}+\cdots+A_{0}=\mathrm{o}, \quad A_{n}=\mathrm{I}
$$

aux racines $\zeta_{v}, v=1,2, \ldots, n,\left|\zeta_{1}\right| \leqq\left|\zeta_{2}\right| \leqq \cdots \leqq\left|\zeta_{n}\right|$, ne sont donnés que les modules des $A_{v}$. Entre quelles limites varie alors le module $\left|\zeta_{\nu}\right|$ de la vième racine de $F(z)$ ?

Si par exemple chaque $\zeta_{v}$ varie dans une couronne circulaire d'épaisseur relative bornée uniformement il existe $n$ nombres positifs $Q_{\nu}, \nu=\mathrm{I}, \ldots, n$, tels que pour une constante $\lambda_{n}$ ne dépendant que de $n$, chaque $\zeta_{\nu}$ reste dans la couronne

$$
\frac{Q_{v}}{\lambda_{n}} \leqq\left|\zeta_{\nu}\right| \leqq \lambda_{n} Q_{\nu}
$$

Il est facile à voir qu'alors, s'il existe un procédé plus ou moins simple permettant de calculer les $Q_{v}$ en partant des valeurs des $\left|A_{\mu}\right|$, le problème $\mathrm{A} \alpha$.) peut 
être considéré comme résolu. En effet, en calculant les nombres $Q_{\nu}=Q_{v}^{(k)}$ pour la $k$-ième transformée de Graeffe $f_{k}(z)$ de $f(z)$ on a évidemment

$$
\begin{aligned}
& \frac{\mathrm{I}}{\lambda_{n}} Q_{v}^{(k)} \leqq\left|\zeta_{v}\right|^{*^{k}} \leqq \lambda_{n} Q_{v}^{(k)} \\
& \lambda_{n}^{-2^{-k}} \leqq \frac{\left|\zeta_{v}\right|}{Q_{v}^{(k) 2^{-k}}} \leqq \lambda_{n}^{2^{-k}}
\end{aligned}
$$

et l'on obtient non seulement la suite $\sqrt[2^{k}]{\left(Q_{v}^{(k)}\right.}$ convergeant vers $\left|\zeta_{v}\right|$, mais aussi une évaluation de la différence

$$
\left|\sqrt[2^{k}]{Q_{v}^{(k)}}-\right| \zeta_{v}||
$$

Or, nous allons démontrer dans ce mémoire qu'en effet les $\zeta_{v}$ varient dans une couronne circulaire dont l'épaisseur relative est par exemple $<(n+1)^{2}$.

Quant aux nombres $Q_{v}$, on peut les déterminer en utilisant le diagramme de Newton du polynôme $F(z)$. - Et c'est en introduisant le diagramme de Newton dans la théorie de la méthode de Graeffe qu'on parricnt à résoudre les problèmes $\mathbf{A} \alpha.), \mathbf{A} \beta.), \mathbf{A} \gamma)$, B.), C.).

Ce diagramme a été utilisé par Newton et surtout par Puisenx dans la théorie des fonctions algébriques d'une variable. Plus récemment $M$. G. Dumas ${ }^{1}$ a appliqué l'idée de ce diagramme pour formuler quelques théorèmes de décomposition dans la théorie "formelle» des fonctions algébroides et les théorèmes analogues dans la théorie des nombres algébriques $p$-adiques. En poursuivant ces idées de M. Dumas, nous avons généralisé ses résultats pour la théorie générale des »valuations» (Bewertungen), et en particulier pour les fonctions algébriques de séries de Dirichlet". De l'autre côté M. Hadamard ${ }^{3}$ a, dès 1892 , appliqué le diagramme de Newton à la théorie de la croissance des fonctions entières et en a tiré quelques conséquences sur les zéros des fonctions entières qui servaient de base à sa théorie des fonctions entières.

En ce qui concerne le présent mémoire, la conséquence la plus importante de la théorie du diagramme de Newton consiste dans le fait suivant:

1 Dumas (1) et (2) pp. 214-217. Le théorème de M. Duras sur "ladaition des diagrammes" est dans le cas spécial des fonctions algébriques d'une variable naturellement une conséquence immédiate des théorèmes de Puiseux. Néanmoins l'énoncé de M. Dumas constitue même dans ces cas un progrès très important et féconde par le point de vae original dont il est l'expression.

2 OSTRowskr (I) pp. I $2 \mathrm{I}$-I 26.

3 HADAMARD (I).

14-39615. Acta mathematica. 72. Imprimé le 1 mars 1940. 
Supposons, pour simplifier, que $A_{0} \neq 0, A_{n} \neq$ o, alors $F(z)$ possède sa $\gg m a$ jorante de Newton" $\mathfrak{M}_{F}(z)$ uniquement déterminée, c'est-à-dire un polynôme

tel que

$$
\mathfrak{M}_{F}(z) \equiv \sum_{\nu=0}^{n} T_{\nu} z^{v}
$$

I.) $\left|A_{\nu}\right| \leqq T_{\nu}, \quad \nu=0, \ldots, n$;

2.) $\quad R_{v}=\frac{T_{v-1}}{T_{v}} \leqq \frac{T_{v}}{T_{v+1}}=R_{v+1}, \quad v=\mathrm{I}, \ldots, n-\mathrm{I}$;

3.) si un second polynôme $\sum_{v=0}^{n} T_{v}^{\prime} z^{v}$ possède les propriétés I.) et 2.) on a

$$
T_{v} \leqq T_{v}^{\prime}, \quad v=0, \ldots, n
$$

Les nombres $R_{v}$ seront appelés dans ce qui suit les inclinaisons numériques du diagramme de Newton de $F(z)$, respectivement du polynôme $F(z)$.

Or, on peut poser $Q_{v}=R_{v}$.

Plus précisément nous démontrons dans le théorème VII - le premier théorème. fondamental - qu'on a pour chaque $\nu$

$$
\left|\zeta_{v}\right| \geqq\left(\mathrm{I}-\left(\frac{\mathrm{I}}{2}\right)^{\frac{1}{\nu}}\right) R_{v}
$$

où la constante I $-\left(\frac{\mathrm{I}}{2}\right)^{\frac{1}{v}}$ est $"$ la meilleure» pour chaque $\nu$. On en tire tout de suite comme borne supérieure de $\left|\zeta_{v}\right|$ :

$$
\frac{R_{v}}{\mathrm{I}-\left(\frac{\mathrm{I}}{2}\right)^{n-v+1}}
$$

Du reste nous démontrons l'inégalité indiquée non seulement pour polynômes, mais aussi pour les fonctions entières et même pour les séries entières.

Une inégalité analogue pour le produit $\left|\zeta_{1} \ldots \zeta_{v}\right|$ des premières $v$ racines d'une fonction entière:

$$
\left|\zeta_{1} \ldots \zeta_{v}\right| \geqq \frac{\mathrm{I}}{v+\mathrm{I}} R_{1} \ldots R_{v}
$$

a été déduit par M. Hadamard dans le mémoire cité par une déduction autant ingénieuse que compliquée ${ }^{1}$. Nous devons à une communication de M. G: Pólya

1 HADAMARD (I) pp. I94-20I. 
une démonstration extrèmement simple, basée sur la formule de Jensen, d'une inégalité un peu plus précise:

$$
\left|\zeta_{1} \ldots \zeta_{\nu}\right| \geqq \sqrt{\frac{v^{\nu}}{(\nu+\mathrm{I})^{\nu+1}}} R_{1} \ldots R_{\nu} .
$$

Nous tenons à exprimer ici notre reconnaissance à M. Pólya pour la permission de reproduire sa belle démonstration dans notre mémoire.

Pour poursuivre la discussion du problème énoncé plus haut, l'introduction des nombres

$$
D_{v}=\frac{R_{v+1}}{R_{v}}, \quad \nu=\mathrm{I}, \ldots, n-\mathrm{I}
$$

est indispensable. Nous appelons ces nombres les déviations du diagramme de Newton de $F(z)$, respectivement du polynôme $F(z)$. Alors on peut annoncer comme condition suffisante pour $\left|\zeta_{m}\right|<\left|\zeta_{m+1}\right|$ l'inégalité

$$
D_{m}>9^{1} \text {. }
$$

Cette condition n'est, naturellement, pas nécessaire, mais si l'on a, pour un polynôme $f(z),\left|\zeta_{m}\right|<\left|\zeta_{m+1}\right|$, il est facile à voir que la condition $D_{m}>9$ est satisfaite pour les transformées de Graeffe $f_{k}(z)$ à partir d'un $k$.

Avec ce résultat le problème $A \beta$.) peut être considéré comme résolu.

Enfin, une solution du problème $\Delta \gamma$.), théoriquement complète est contenu dans la relation (5), mais pour le but pratique on emploira plutôt les évaluations dont nous parlons plus bas en connexion avec le problème $\mathrm{B}$.)

Quant au problème B.) — celui de détermination complète des $\zeta_{v}$ (c'est-à-dire avec leurs arguments) - on trouve déjà dans le mémoire de Graeffe des méthodes permettant dans des cas très généraux le calcul des arguments des $\zeta_{v}$ dès que les $\left|\zeta_{v}\right|$ sont connus.

1 Cette condition a déjà été utilisée par M. HADamaRD (2) dans la théorie des fonctions entières et des séries de LAURENT pour déduire une relation qui montre qne sous cette condition le terme du rang $m$ de la série domine sur une certaine circonférence sur l'ensemble de tous les autres termes de la série. Il en déduit une méthode de construction d'une fonction entière de genre infini qui ne rentre pas dans les cas d'exception que comporte le théorème de M. PICARD.

M. VAliRon (I) pp. I6-i 8 reprend la méthode de M. HADAMARD et en déduit que si pour une fonction entière on a pour un $m: D_{m}=\frac{R_{m+1}}{R_{m}}>9$ la fonction possède exactement $m$ zéros dans le cercle $|z|<\sqrt{R_{m} R_{m+1}}$. Il résulte de ce beau résultat de M. VAIIron que, les racines de la fonction étant rangées dans l'ordre des modules croissants, le module de la $m$-ième racine est différent du module de la racine suivante. Dans le cas des polynômes, c'est exactement la relation $\left|\zeta_{m}\right|<\left|\zeta_{m+1}\right|$. 
Dans le cas le plus général on peut réduire le calcul des $\zeta_{v}$ à celui des $\zeta_{v}^{2 k}$ en utilisant un artifice de Carvallo. Il suffit done de calculer toutes les racines d'une transformée $f_{k}(z)$ de Graeffe convenablement choisie. Or, c'est ici que le point de vue de décomposition de $f_{k}(z)$ en polynômes partiels, développé par Dandelin dans son mémoire original, devient très important.

Supposons que les $n$ racines $\zeta_{v}$ de $f(z)$ soient toutes $\neq 0$ et se décomposent en un certain nombre de groupes:

$$
\begin{gathered}
x_{1}, \ldots, x_{\alpha} ; y_{1}, \ldots, y_{\beta} ; \ldots ; z_{1}, \ldots, z_{\gamma}^{\prime} ; \\
\alpha+\beta+\cdots+\gamma=n,
\end{gathered}
$$

tels que les modules de tous les $x$ ne sont pas très différents entre eux tandis qu'ils sont essentiellement plus petits que les modules des $y$. De même les modules des $y$ ne sont pas sensiblement différents entre eux tandis qu'ils sont essentiellement plus petits que les modules des racines du groupe suivant, etc.

Alors, si $k$ est suffisemment élevé, les puissances $x_{1}^{2^{k}}, \ldots, x_{\alpha}^{2^{k}}$ des racines du premier groupe satisfont - avec une précision de plus en plus croissante - à l'équation

$$
a_{0}^{(k)}-a_{1}^{(k)} z+\cdots+a_{\alpha}^{(k)}(-\mathrm{I})^{\alpha} z^{\alpha}=0
$$

formée de l'ensemble des termes correspondants de $f_{k}(z)$. De même les puissances $y_{1}^{2^{k}}, \ldots, y_{\beta}^{2^{k}}$ satisfont à l'équation

$$
a_{\alpha}^{(k)} z^{\alpha}-a_{\alpha+1}^{(k)} z^{\alpha+1}+\cdots+a_{\alpha+\beta}^{(k)}(-\mathrm{I})^{\beta} z^{\alpha+\beta}=0
$$

et le fait analogue étant valable pour chaque groupe de racines, les puissances $z_{1}^{2^{k}}, \ldots, z_{\gamma}^{2^{k}}$ des racines du dernier groupe satisfont à l'équation

$$
a_{n-\gamma}^{(k)} z^{n-\gamma}-a_{n-\gamma+1}^{(k)} z^{n-\gamma+1}+\cdots+a_{n}^{(k)}(-\mathrm{I})^{\gamma} z^{n}=0 .
$$

Dandelin ${ }^{1}$ établit le fait énoncé en supposant que les modules des racines de chaque groupe sont »infiniment petits» par rapport aux modules des racines du groupe suivant. Alors la décomposition a lieu déjà pour le polynôme $f_{0}(z)$.

Sans tenir compte de cet énoncé, les calculateurs, en utilisant la méthode de Graeffe, retrouvaient le même fait pour ainsi dire »expérimentalement: en remarquant que si l'on fait le calcul avec un certain nombre de chiffres significatifs, les transformées $f_{k}(z)$, à partir d'une valeur de $k$, se décomposent en "fragments» tels que dans le passage aux transformées d'ordre supérieur les

1 Dandelin (1) pp. $5 \mathbf{I}-57$. 
coefficients appartenant aux fragments différents n'exercent plus aucune influence les uns sur les autres, de sorte qu'on n'a qu'à calculer les transformées suivantes pour chaque fragment indépendamment.

Or, ces faits trouvent une expression précise et rigoureuse dans notre troisième théòème fondamental, qui permet de résoudre complètement le problème $\mathrm{B}$.). Supposons que pour le polynôme $F(z)$ deux déviations $D_{m}, D_{l}, m>l$, soient $\geqq \mathrm{I} 8,7$. Alors à l'ensemble des termes correspondants de $F(z)$ :

$$
\sum_{y=l}^{m} A_{v} z^{v}=A_{m} z^{l} G_{0}(z)
$$

correspond un facteur $Q(z)=z^{m-l}+\cdots$ de $F(z)$ de degré $m-l$, tel que les coefficients de la différence $Q(z)-G_{0}(z)$ sont très petits par rapport aux coefficients correspondants de la majorante newtonienne de $G_{0}(z)$ :

$$
Q(z)-G_{0}(z) \ll \delta \mathfrak{M}_{G_{0}}(z)^{1}
$$

Ici $\delta$ converge vers o si $D_{m}$ et $D_{l}$ croissent vers l'infini. On a p. ex.

$$
\delta<\frac{5}{\operatorname{Max}\left(D_{m}, D_{l}\right)-10}
$$

Si l'on veut décomposer $F(z)$ en deux fragments seulement, on peut remplacer la constante I8,7 par 13,5. En renvoyant pour d'autres précisions aux énoncés des théorèmes XXVI, XXVII, relevons seulement que pour $m-l=\mathbf{I}$, c'est-à-dire quand il s'agit d'isoler et de calculer une seule racine de $F(z)$, la constante 18,7 peut être remplacée par la racine positive $M_{0}=6,357355627 \ldots$ de l'équation

$$
M^{3}-5 M^{2}-8 M-4=0
$$

de sorte que si l'on a $D_{m}>M_{0}, D_{m-1}>M_{0}$, il y a une et une seule racine $\zeta_{0}$ de $F(z)$ pour laquelle on a p. ex.:

$$
\left|\frac{\zeta_{0}}{A_{m-1} / A_{m}}+\mathrm{I}\right| \leqq \frac{5,84 \cdots}{\operatorname{Max}\left(D_{m}, D_{m-1}\right)} .
$$

C'est une partie de notre deuxième théorème fondamental (théorème XVI).

Ces résultats peuvent être précisés encore, si toutes les déviations de $F(z)$ sont suffisamment grandes. Dans ce cas il suffit déjà que toutes les déviations

1 La relation $A(z) \ll B(z)$ exprime suivant PoINCARḱ que $A(z)$ est majoré par $B(z)$, c'est-à-dire que chaque coefficient de $A(z)$ est en module $\leqq$ que le coefficient correspondant de $B(z)$. 
de $F(z)$ sont $\geqq v_{n}^{2}$ où les valeurs de $v_{n}^{2}$ sont $\geqq 4$ et croissent avec $n$ en convergeant vers $4,8105^{8} \ldots 1$.

En remplaçant un facteur $Q(z)$ de $F(z)$, en vertu de (6), par $G_{0}(z)$ on modifie naturellement les racines de $Q(z)$; mais dans ce cas le théorème, que les racines d'un polynôme sont des fonctions continues de ses coefficients, ne suffit pas pour contrôler l'influence de $\delta$ dans la formule (6) sur les variations des racines. En effet, $\delta$ dans (6) est une mesure de l'erreur relative des coefficients de $Q(z)$ tandis que dans le théorème cité il s'agit des erreurs absolues.

D'autre part seule la considération des erreurs relatives est conforme aux habitudes dẹ calculateurs qui se bornent généralement à un nombre fixe de chiffres pour chaque coefficient sans tenir compte de la grandeur absolue de ces coefficients.

Or, le théorème suivant sur la continuité des racines des équations algébriques se rapporte aux erreurs relatives:

Il existe pour chaque degré $k$ une fonction $\eta(\delta)=\eta_{k}(\delta)$ positive pour $\delta>0$ et tendant vers o avec $\delta$, telle que, si l'on a (6) pour deux polynômes $G_{0}(z)$ et $Q(z)$ du degré $k$, on a en désignant leurs racines dans l'ordre convenable respectivement par $x_{x}$ et $y_{x}$ :

$$
\left|\frac{y_{x}}{x_{x}}-\mathrm{I}\right|<\eta(\delta) ; \quad \quad x=\mathrm{I}, \ldots k
$$

On peut poser par exemple, si $\mathrm{I} \sigma k \delta^{\frac{1}{k}} \leqq \mathrm{I}$ :

$$
\eta(\delta)=\mathrm{I} 5 k \delta^{\frac{1}{k}}
$$

(quatrième théorème fondamental). C'est ce dernier théorème qui permet évidemment de résoudre complètement le problème C.) énoncé plus haut, bien que le module de continuité relative donné par $\eta(\delta)$ apparait naturellement trop faible pour les buts pratiques. Or, comme nous montrons an No. 75 sur un exemple, assez artificiel, il est vrai, l'ordre de $\eta(\delta)$ peut être en effet atteint.

De l'autre côté, en étudiant dans les Nos. 83-86 la propagation des erreurs des coefficients dans le passage d'une transformée de Graeffe à la transformée suivante, nous obtenons des évaluations également très désavantageuses, mais qui ne peuvent pas apparamment être essentiellement améliorées dans le cas le plus général.

1 Le résultat correspondant pour les séries entières avec $4,8 \mathrm{\ldots} \ldots$ au lieu de $v_{n}^{2}$ se trouve déjà chez M. Valiron, VaLIRoN (I), pp. I6-i8. 
Il s'agit ici du reste d'un phénomène qui se présente souvent et très naturellement dans les applications de l'analyse. Dès qu'on cherche à embrasser tous les cas possibles, les évaluations des erreurs deviennent pratiquement inutilisables.

Or, c'est naturellement le théoricien qui doit changer la manière de poser la question. Ce qu'il cherche d'abord, ce sont les vévaluations a priorì dans lesquelles on n'utilise que très immédiatement les données du problème. Ce dont le calculateur a surtout besoin, ce sont les "évaluations a posteriori» dans lesquelles on utilise les résultats du calcul approximatif, une fois effectué. Et c'est en se plaçant à ce point de vue qu'on parvient dans la plupart des cas aux résultats d'utilité pratique immédiate.

Du reste ce ne sont que les deux derniers $\$ \S$ du dernier chapitre du présent mémoire qui sont consacrés explicitement à la méthode de Graeffe. Tout le reste de ce mémoire est consacré aux théorèmes généraux.

Dans cette recherche il n'était pas du tout nécessaire de nous borner aux polynômes. La plupart des résultats des trois premiers chapitres sont valables pour les fonctions entières et même pour les séries entières et (sauf les résultats du second chapitre) celles de Laurent et nous les avons développés dès la commencement dans toute leur généralité.

Ceci n'allait naturellement pas sans quelques inconvénients. Tout d'abord on a dû ajouter parfois quelques développements additionnels, les résultats dont il s'agissait étant plus faciles à démontrer pour les polynômes. Dans ce cas, le lecteur qui ne s'intéresse qu'aux polynômes n'aura qu'à laisser de côté ces parties du mémoire.

D'un autre côté, dans la théorie des fonctions algébriques et ses généralisations on écrit généralement un polynôme du degré $n$ dans la forme suivante: $A_{0} z^{n}+$ $+A_{1} z^{n-1}+\cdots+A_{n}$ et construit le diagramme de Newton en faisant correspondre $v$ au terme $A_{v} z^{n-v}$ comme abscisse. Ceci présente quelques avantages si l'on n'a à faire qu'aux polynômes, mais n'est pas immédiatement applicable aux séries de puissances. C'est pour ne pas utiliser deux constructions différentes du diagramme, que nous nous sommes bornés à une seule construction du diagramme exposé dans le mémoire. Du reste, si l'on ne considère que les polynômes, il est très 
facile d'apporter à nos résultats et démonstrations les modifications nécessitées par l'emploi du diagramme de Newton dans sa deuxième forme ${ }^{1}$.

Enfin, quant aux méthodes de démonstration, les parties du mémoire qui se rapportent aux polynômes n'utilisent pas généralement la théorie des fonctions, le théorème de Rouché dont nous faisons souvent usage dans le chapitre III étant une conséquence immédiate du théorème classique (et démontrable algébriquement) sur la continuité des racines. Seulement dans les démonstrations des théorèmes VII et XV et celle du théorème XXIII la théorie des fonctions intervient essentiellement. C'est pourquoi nous avons ajouté aux endroits correspondants des démonstrations purement algébriques des théorèmes VII et XV, mais avec des constantes un peu plus faibles: pour le théorème VII en remplaçant $\mathrm{I}-\left(\frac{\mathrm{I}}{2}\right)^{\frac{1}{p}}$ par $\left(\frac{3}{2}\right)^{\frac{1}{p}}-\mathrm{I}$ et pour le théorème $\mathrm{XV}$ en remplaçant $\sqrt{\frac{(p+\mathrm{I})^{p+1}}{p^{p}}}$ par $2 p+$ I. Quant au théorème XXIII, il peut être déduit, avec des constantes un peu plus faibles, du troisième théorème fondamental.

La disposition du présent mémoire est la suivante: Dans le premier chapitre sont traitées les définitions du diagramme et de la majorante newtonienne. En particulier dans le $\$ 2$ nous développons la condition nécessaire et suffisante pour l'existence $d u$ diagramme de Newton d'une série de Laurent. Dans le $\$ 4$ de ce chapitre sont rassemblées différentes propriétés du diagramme, des inclinaisons numériques et des déviations dont nous avons à faire usage dans la suite.

Le chapitre II est consacré aux développements daus lequel les inclinaisons numériques, mais pas les déviations, jouent un rôle fondamental. Dans le $\$ 5$ le premier théorème fondamental est démontré pour le cas le plus général des séries de puissances et un énoncé un peu plus faible de ce théorème pour le cas des polynômes par une méthode purement algébrique.

Dans le dernier No. de ce $\S$ nous déterminons tous les cas où dans les relations du premier théorème fondamental le signe d'égalité est valable.

Dans le $\$ 6$ différentes bornes pour les modules des racines sont déduites du premier théorème fondamental. En particulier, nous déduisons l'existence

${ }^{1}$ D'ailleurs on pourrait aussi construire le diagramme en prenant $\lg \left|A_{\nu}\right|$ comme ordonnée et enveloppant les points représentatifs par une ligne brisée convexe d'en haut, ce qui présenterait peut-être quelques avantages. Du reste on obtient toutes ces différentes formes du diagramme de NEwToN d'une quelconque d'elles par symétrie ou bien par rapport à l'axe des $x$ ou bien par rapport à une parallèle à l'axe des $y$. 
d'une constante $c_{n}$ qui est le nombre le plus petit $>$ I avec la propriété que si dans une équation du degré $n$ les arguments des coefficients varient arbitrairement, les modules des coefficients demeurant fixes, la $v$-ième racine de ce polynôme varie dans une couronne circulaire autour de l'origine d'épaisseur relative $c_{n}$, pour chaque $v=\mathrm{I}, \ldots, n$. Nous démontrons en particulier $c_{n}<$ $0,73(n+\mathrm{I})^{2}$. Quant à la détermination complète de $c_{n}$ en fonction de $n$, nous déterminons seulement $c_{2}=\mathrm{I}+\sqrt{2}$. Enfin nous déduisons la relation $\frac{\lim }{n} \geqq \frac{c_{n}}{\pi}$. De l'autre côté on peut se poser le problème de déterminer les meilleures bornes de $\frac{\zeta_{v}}{R_{v}}$ non pas pour un $v$ donné, mais si le degré $n$ de l'équation reste fixe. Nous donnons une discussion complète de ce problème pour les cas $n=2$ et 3 .

Le problème de la détermination complète de ces bornes ainsi que de $c_{n}$ pour $n$ quelconque reste ouvert.

Au \& suivant nous déterminons d'abord une borne supérieure exacte pour le quotient

$$
\frac{\left|\zeta_{1} \ldots \zeta_{p}\right|}{R_{1} \ldots R_{p}}
$$

les racines $\zeta_{v}$ de $F(z)$ étant rangées dans l'ordre des modules croissantes. Quant à la borne inférieure nous reproduisons la récente démonstration de Pólya de l'inégalité d'Hadamard-Pólya et démontrons par une méthode purement algébrique que $\frac{I}{2 p+I}$ est une telle borne.

Le chapitre III est consacré aux théorèmes de décomposition dans lesquelles interviennent les déviations.

Dans le $\S 8$ de ce chapitre nous démontrons le deuxième théorème fondamental qui permet d'isoler une seule racine de l'équation si deux déviations consécutives sont suffisemment grandes. Dans l'énoncé de ce théorème nous avons surtout tenu à abaisser les bornes des déviations autant que possible. La démonstration peut naturellement être considérablement simplifiée si l'on utilise p. ex. la borne 7 au lieu de $M_{0}$.

Dans le $\$ 9$ nous déterminons les bornes correspondantes $v_{n}^{2}$ sous l'hypothèse que toutes les déviations d'un polynôme du degré $n$ sont $\geqq v_{n}^{2}$.

Dans les $\S \S$ Io et I I nous considérons le cas où une déviation $D_{p}$ est $>9$. On obtient dans ce cas l'existence d'une couronne circulaire qui ne contient pas de racines. En utilisant ce résultat, nous obtenons pour le cas où deux déviations

15-39615. Acta mathematica. 72. Imprimé le 1 mars 1940. 
$D_{q}, D_{q^{\prime}}, q>q^{\prime}$, sont $\geqq 9$ une couronne circulaire contenant exactement $q-q^{\prime}$ racines et déduisons au moyen du théorème de Jensen une évaluation assez exacte pour le produit des modules de ces racines.

Dans le $\S$ i2 nous traitons le problème de déterminer autant que possible le diagramme du produit des deux polynômes aux diagrammes connus. A ce problème se rapportent pour le cas des fonctions algébriques d'une variable et celui des nombres algébriques $p$-adiques quelques beaux résultats de $M$. G. Dumas ${ }^{1}$ qui a montré qu'on obtient alors le diagramme du produit pour ainsi dire par addition géométrique des côtés des diagrammes des facteurs, ordonnés convenablement - c'està-dire que dans les cas considérés par M. Dumas les inclinaisons numériques des facteurs se retrouvent sans modification dans le produit. Le même reste encore vrai dans le cas le plus général des "valuations» (Bewertungen) non-archimédiennes ${ }^{2}$. Ces résultats ne restent plus complètement exacts dans le cas considéré ici, mais si les inclinaisons numériques d'un des deux facteurs sont petites par rapport aux inclinaisons numériques du second, les inclinaisons numériques de ces deux facteurs se multiplient dans le passage au produit par des facteurs voisins de un. C'est ce résultat qui, précisé convenablement, est utilisé dans la démonstration $d u$ troisième théorème fondamental. Ce théorème est démontré dans le $\$$ I 3 pour le cas d'un polynôme. Par un passage à la limite on en déduit au $\$ 14$ le théorème correspondant pour le cas le plus général des séries de Laurent.

Au $\S$ suivant nous déduisons du troisième théorème fondamental quelques conséquences relatives à la décomposition d'une série de Laurent dans un produit infini, s'il y a une suite infinie de déviations $\geqq 18,7$.

Dans ce même $\$$ nous démontrons ensuite que si les coefficients de la série de Laurent sont des fonctions régulières dans certaines régions et si les déviations correspondantes restent $\geqq 18,7$ dans ces régions, les coefficients des facteurs peuvent aussi être choisis de manière à être réguliers dans la même région. C'est un résultat de très grande généralité, il contient p. ex. en le généralisant considérablement le célèbre »théorème de préparation» de Weierstrass.

Le chapitre IV est consacré à l'étude des questions qui se rattachent à la continuité des racines des équations algébriques. Nous commencons dans le $\$$ i 6 par préciser le théorème connu sur la continuité des racines d'un polynôme du degré $n$ en démontrant que ces racines satisfont comme fonctions des coefficients

1 DuMAs (1) et (2) pp. $214-217$.

2 Ostrowski (I) pp. I 2 I - 26. 
à une condition de Lipschitz d'ordre $\frac{\mathrm{I}}{n}$. Ensuite nous démontrons le quatrième theorème fondamental sur la continuité relative des racines d'un polynôme et finissons ce $\S$ par quelques remarques sur la possibilité d'améliorer les évaluations de ce théorème. En particulier nous traitons dans le No. 77 en détail le cas d'une équation quadratique.

Dans le $\S$ I 7 nous appliquons les résultats généraux du mémoire à la méthode de Graeffe en ce qui concerne la détermination des modules des racines. La deuxième partie de ce $\S$ (Nos. $83-86$ ) est consacré à l'étude de la propagation des erreurs dans le calcul des transformées de Graeffe.

Enfin, dans le dernier $\S$ du mémoire nous passons en revue les différentes méthodes qui peuvent être utilisées pour le calcul des arguments, traitons aux Nos. 92-94 trois exemples plus ou moins caractéristiques et finissons dans le No. 95 par quelques remarques sur la possibilité de simplifier l'application du procédé de Newton aux valeurs acquises par la méthode de Graeffe ${ }^{1}$.

\section{CHAPITRE I. Le diagramme de Newton et les séries normales.}

\section{$\S$ I. Les polynômes normaux.}

I. Un polynôme

$(I, I)$

$\sum_{\nu=0}^{n} a_{\nu} z^{v}$

aux coefficients réels $\geqq 0$ est dit normal, si pour $\nu<\mu, a_{\nu}>0, a_{\mu}>0$ on a toujours $a_{x}>0(x=\nu+\mathrm{I}, \ldots, \mu-\mathrm{I})$ et
$(\mathrm{I}, 2)$
$a_{v}^{2} \geqq a_{p-1} a_{v+1}$
$(v=\mathrm{I}, \ldots, n-\mathrm{I})$.

Il résulte de la définition que, si $a_{n_{0}}, a_{n_{1}}$ sont le premier resp. le dernier coefficient de $(\mathrm{I}, \mathrm{I})$ différent de o, on a $a_{v}>$ o pour $n_{0} \leqq \nu \leqq n_{1}$.

Plus généralement une série de puissances

1 Les idées générales développées dans le présent memoire ont été esquissées dans une conférence tenue le 23 mars 1938 à l'Institut des hautes études de Belgique. Quelques résultats de ce mémoire ont été publiés 1938 et I939 dans les C. R. de l'académie des sciences de Paris. Cf. Ostrowski (2) et (3). 
$(1,3)$

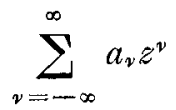

aux coefficients réels $\geqq 0$ est dite normale, si l'on a pour chaque $\nu$

$$
a_{v}^{2} \geqq a_{\nu-1} a_{v+1}
$$

et chaque fois que pour $\nu<\mu, a_{v}>0, a_{\mu}>0$, on a aussi $a_{x}>0$ pour $x=\nu+\mathrm{I}$, ..., $\mu-$ I. Si l'on a même $a_{v}^{2}>a_{v-1} a_{v+1}$ chaque fois que $a_{v-1} a_{v} a_{v+1}$ est positif, nous appellerons $(\mathrm{I}, 3)$ normale au sens étroit.

2. Chaque polynôme

$$
f(z)=A_{0}+A_{1} z+\cdots+A_{n} z^{n}
$$

possède une infinité de polynômes normaux comme majorantes.

Nous allons montrer qu'il y a parmi les majorantes normales de $f(z)$ une

$$
\mathfrak{M}_{f}(z)=T_{0}+T_{1} z+\cdots+T_{n} z^{n}
$$

dont tous les coefficients sont minimum, c'està-dire telle que si le polynôme (r, I) est une majorante normale quelconque de $f(z)$ on a

$$
a_{v} \geqq T_{v}
$$$$
(\boldsymbol{v}=\mathrm{o}, \mathrm{I}, \ldots, n)
$$

Pour construire le polynôme $\mathfrak{M}_{f}(z)$ on peut utiliser le procédé suivant: Supposons que $A_{0} A_{n} \neq 0$, ce qu'on peut faire sans restreindre la généralité; alors à chaque terme $A_{v} z^{v}, A_{v} \neq$ o, de $f(z)$ correspond le point représentatif $P_{v}: x=v$, $y=-\lg \left|A_{v}\right|$. Enveloppons les points $P_{v}$ par une ligne brisée $D$ convexe d'en bas de sorte que $I^{\circ}$ ) chaque sommet de $D$ est situé dans un des $P_{v}$ et que $2^{\circ}$ ) tous les autres points $P_{v}$ sont situés sur $D$ ou au dessus. En particulier $D$ commence au point $P_{0}$ et se termine au point $P_{n}$. (Voire fig. I au no. Io).

La ligne $D$ sera appelée le diagramme de Newton du polynome $f(z)$.

3. Soit $x_{v}$ l'ordonnée du point de $D$ correspondant à l'abscisse $\nu(0 \leqq \nu \leqq n)$. Soit $D^{*}$ une ligne convexe d'en bas telle que tous les points $P_{v}$ sont situés sur $D^{*}$ ou au dessus. Alors, comme $D$ consiste en segments rectilignes joignant les points $P_{v}$ entre eux, chaque point de $D$ sera situé sur $D^{*}$ ou au dessus.

Posons maintenant

$$
T_{\nu}=e^{-x_{\nu}} \neq 0 \quad(0 \leqq \nu \leqq n) ; T_{v}=0 \quad(\nu>n),
$$


alors je dis qu'on a

$$
T_{v}^{2} \geqq T_{v-1} T_{v+1}
$$

En effet cette relation se réduit à l'inégalité $x_{v} \leqq \frac{\mathrm{I}}{2}\left(x_{v+1}+x_{v-1}\right)$, où $v=\mathrm{I}, \ldots$, $n$ - I, qui résulte immédiatement du caractère convexe de la ligne $D$.

Donc le polynôme $(2,2)$ formé avec ces $T_{v}$ est normal.

4. Soit (I, I) un polynôme normal avec $a_{0} a_{n} \neq 0$. Marquons les points représentatifs $P_{v}^{\prime}(o \leqq \nu \leqq n)$ correspondant aux différents termes de ce polynôme.

Alors la ligne brisée

$$
P_{0}^{\prime} P_{1}^{\prime} \ldots P_{v}^{\prime} \ldots P_{n}^{\prime}
$$

est convexe d'en bas, comme il résulte immédiatement de la relation ( $\mathrm{I}, 2)$. Donc cette ligne est le diagramme de Newton $D^{\prime}$ correspondant au polynôme (I, I). Or, supposons que le polynôme (I, I) soit une majorante normale de $f(z)$. Alors il résulte de $\left|A_{v}\right| \leqq a_{v}$ que chacun des points $P_{v}$ est situé sur la ligne $D^{\prime}$ ou audessus.

Donc, d'après ce que nous avons dit plus haut, chaque point de $D$ est situé sur $D^{\prime}$ ou au dessus. Donc en particulier

$$
x_{v} \geqq-\lg a_{v}, T_{v} \leqq a_{v} \quad(v=0, \ldots, n)
$$

et nous avons démontré le théorème

I. Il existe une majorante normale $(2,2)$ du polynôme $(2, \mathrm{I})$ telle que, si $(\mathrm{I}, \mathrm{I})$ est une majorante normale quelconque de $(2,1)$, on $a(2,3)$.

Nous appelons le polynôme $(2,2)$ la majorante newtonienne de $f(z)$.

On a évidemment $\left|A_{0}\right|=T_{0},\left|A_{n}\right|=T_{n}$.

5. II. Soient $z_{1}, \ldots, z_{n}$ les racines du polynôme

$$
f(z)=A_{n} z^{n}+\cdots+A_{0}, A_{0} A_{n} \neq \mathrm{o}
$$

prises avec lewrs multiplicités correspondantes. Alors, si $(2,2)$ est la majorante newtonienne de $f(z)$, on a

$$
\frac{T_{v}}{T_{n}} \leqq C_{v}
$$

où

$$
\prod_{p=1}^{n}\left(z+\left|z_{y}\right|\right)=C_{n} z^{n}+\cdots+C_{0} .
$$


En effet, le polynôme $(5,2)$ est normal, ce qui résulte des inégalités de Newton $C_{v}^{2} \geqq C_{v-1} C_{v+1}$. Donc le produit du polynôme $(5,2)$ par $\left|A_{n}\right|=T_{n}$ est une majorante normale de $f(z)$ et $(5, \mathrm{I})$ découle immédiatement du théorème $\mathrm{I}$, C. Q. F. D.

\section{$\S$ 2. Le diagramme d'une série de Laurent.}

6. Considérons maintenant plus généralement l'expression

$$
f(z)=\sum_{v=-\infty}^{+\infty} a_{v} z^{\nu} .
$$

Le point représentatif d'un terme $a_{v} z^{v},\left(a_{v} \neq \mathrm{o}\right)$ de $(6, \mathrm{I})$ est

$$
P_{v}: x=\nu, y=\lg \frac{\mathrm{I}}{\left|a_{v}\right|}
$$

De chacun des points $P_{v}$ menons une demi-droite parallèle à la direction des $y$ positifs et désignons par $E_{0}$ l'ensemble des points de ces demi-droites. Soit $E^{*}$ l'enveloppante de $E_{0}$, c'est-à-dire l'ensemble des points de tous les segments de droite joignant les points de $E_{0}$ deux à deux et des points limites de ces points. Soit $E$ l'ensemble des points frontières de $E^{*}$.

En projettant l'ensemble $E$, s'il n'est pas vide, sur l'axe des $x$ on obtient un intervalle $J$ qui peut se réduire à un seul point, mais pent aussi s'étendre à l'infini dans un ou deux sens.

Supposons que $J$ ne se réduit pas à un seul point. À chaque point de $J$ correspond un point de $E$ dont l'ordonnée est minimum. L'ensemble de ces points est une ligne brisée $D$ convexe d'en bas que nous appelons le diagramme de Newton de $f(z)$.

7. $D$ est caractérisé par les propriétés suivantes:

$\left.I^{\circ}\right)$ Chaque sommet de $D$ est situé dans un des $P_{\nu}$.

$2^{\circ}$ ) Tous les autres $P_{v}$ sont situés sur $D$ ou au dessus.

$\left.3^{\circ}\right)$ Supposons que $D$ contient une demi-droite $L$ issue d'un point $P_{v}=A$. Alors de deux choses une: Ou bien $L$ contient une infinité de points $P_{v}$; ou bien, désignant par $B$ le dernier des points $P_{y}$ sur $L$ et par $L^{\prime}$ la partie de $L$ à partir de $B$, on rencontrera une infinité de points $P_{v}$ en tournant $L^{\prime}$ autour de $B$ de l'angle $\varepsilon$ aussi petit que l'on veut. - (On tourne $L^{\prime}$ dans le sens positif si $L^{\prime}$ va à l'infini à droite; dans le sens négatif, si $L^{\prime}$ va à l'infini à gauche). 
$\left.4^{\circ}\right)$ Si $D$ se réduit à une droite $L$ contenant au moins un point $P_{v}=A$ les deux demi-droites de $D$ issues de $A$ satisfont chacune à la condition $3^{\circ}$ ).

$\left.5^{\circ}\right)$ Si $D$ se réduit à une droite qui ne contient aucun point $P_{v}$, il existe même une suite partielle des $P_{v}$ dont les abscisses convergent vers $+\infty$ ou $-\infty$ et dont les distances de $D$ convergent vers 0 , tandis que pour chaque demi-droite contenu dans $D$ et allant vers $-\infty$ ou $+\infty$ la condition de $3^{\circ}$ est satisfaite.

8. III. Si une série de Taylor $f(z)(6, \mathrm{I})$ possède plus d'un terme $\neq \mathrm{o}\left(a_{\nu}=0\right.$, $\nu<0)$ la condition nécéssaire et suffisante pour qu'elle possède un diagramme de Newton est que son rayon de convergence soit positif.

Démonstration. Si $y=\alpha x+\beta, \alpha \neq 0$ est l'équation d'un quelconque des côtés de $D$ on a

$$
\lg \frac{\mathrm{I}}{\left|a_{\nu}\right|} \geqq \alpha \nu+\beta ; \underline{\lim } \frac{\mathrm{I}}{\sqrt{\left|a_{v}\right|}} \geqq e^{\alpha},
$$

donc le rayon de convergence de $f(z)$ est $\geqq e^{\alpha}$ et positif.

Supposons d'autre part que la série de Taylor $(6, \mathrm{I})$ ait un rayon de convergence positif, c'est-à-dire, que pour deux constantes positives $c, r$, convenablement choisies, on ait

$$
\left|a_{\nu}\right|<e r^{\nu} \quad(\nu=0, I, \ldots)
$$

Alors tous les points $P_{v}$ sont situés au-dessus de la droite $L$

$$
y=x \lg \frac{\mathrm{I}}{\gamma^{*}}-\lg c .
$$

A chacun des points $P_{v}$ correspond un point de $E$ avec l'abscisse $\nu$, et l'intervalle $J$ ne se réduit pas à un seul point.

9. IV. La condition nécessaire et suffisante pour qu'une série de Laurent $(6, \mathrm{I})$ possédant plus d'un terme $\neq$ o possède un diagramme de Newton, est que pour un $z \neq 0$ tous ses termes soient bornés en module dans leur ensemble.

Démonstration. Si $(6, \mathrm{I})$ possède un diagramme de Newton, soit $y=\alpha x+\beta$ la droite contenant un de côtés du diagramme. Alors, puisque aucun des $P_{v}$ n'est situé au dessous de cette droite:

$$
\lg \frac{\mathrm{I}}{\left|a_{\nu}\right|} \geqq \alpha \nu+\beta,\left|\left(e^{\alpha}\right)^{v} a_{\nu}\right| \leqq e^{-\beta}
$$

Donc les termes de $(6, \mathrm{r})$ sont bornés en module pour $z=e^{\alpha}$. 
D'un autre côté, s'il existe un $z \neq 0,|z|=e^{\alpha}$ et une constante positive $c=e^{-\beta}$ telle qu'on a

$$
\left|a_{v} z^{v}\right| \leqq e^{-\beta}
$$

il en résulte $\left|e^{\alpha}\right|^{\nu}\left|a_{\nu}\right| \leqq e^{-\beta}$, donc

$$
\lg \frac{\mathrm{I}}{\left|a_{\nu}\right|} \geqq \alpha \nu+\beta
$$

et aucun des points $P_{y}$ n'est situé au dessous de la droite $y=\alpha x+\beta$. Donc $E$ contient au moins deux points différents: $x=\nu, x=\nu^{\prime}$ et le diagramme de Newton existe,

C. Q. F. D.

En particulier il existe toujours un diagramme de Newton pour une série de Laurent possédant plus d'un terme $\neq 0$, si cette série possède une couronne de convergence.

\section{§ 3. La majorante newtonienne, les inclinaisons numériques et les déviations.}

Io. Si $(6, I)$ est une série normale, tous les points représentatifs $P_{v}$ de ses termes sont situés sur la ligne $D$. -

Soit $a_{n_{0}}$ le premier coefficient de $(6, \mathrm{I})$ qui est $\neq 0$, s'il existe. Dans le cas contraire soit $n_{0}=-\infty$. De même soit $n^{\prime}$ l'indice du dernier coefficient $\neq$ o de $(6,1)$, s'il existe. Dans le cas contraire soit $n^{\prime}=\infty$. Supposons d'abord que $n^{\prime}>n_{0}$.

Soit $\varkappa_{\nu}\left(n^{\prime} \geqq \nu \geqq n_{0}\right)$ l'ordonnée du point de $D$ correspondant à l'abscisse $\nu$. Considérons le segment $\langle\boldsymbol{v}-\mathrm{I}, \boldsymbol{\nu}\rangle$ de l'axe des $x$ et le segment $\boldsymbol{\sigma}_{\boldsymbol{v}}$ de $D$ se projetant sur $\langle\nu-\mathrm{r}, \nu\rangle$. $\sigma_{\nu}$ est le $\nu$-ième segment élémentaire de $D$. La différence $x_{v}-x_{v-1}$ est l'inclinaison logarithmique de $\sigma_{v}$ et la $\nu$-ième inclinaison logarithmique de $f(z)$ resp. de $D$ - le tg de l'angle entre $\sigma_{v}$ et l'axe des $x$. Posons

$$
\begin{gathered}
T_{v}^{\prime}=e^{-x_{v}} \quad\left(n^{\prime} \geqq \nu \geqq n_{0}\right), \quad T_{v}=0 \quad\left(v<n_{0}, v>n^{\prime}\right), \\
R_{v}=e^{x_{v}-x_{v}-1}=\frac{T_{v-1}}{T_{v}} \quad\left(n^{\prime} \geqq \nu>n_{0}\right), R_{n_{0}}=0, R_{n^{\prime}+1}=\infty, \\
D_{v}=\frac{R_{v+1}}{R_{v}} \quad\left(n_{0}<\nu<n^{\prime}\right), \quad D_{n_{0}}=D_{n^{\prime}}=\infty .
\end{gathered}
$$

$R_{v}$ sera appelée la $\nu$-ième inclinaison numérique de $f(z)$ resp. de $D, D_{v}$ est la $\nu$-ième déviation de $f(z)$ resp. de $D$. (Voir fig. I). 


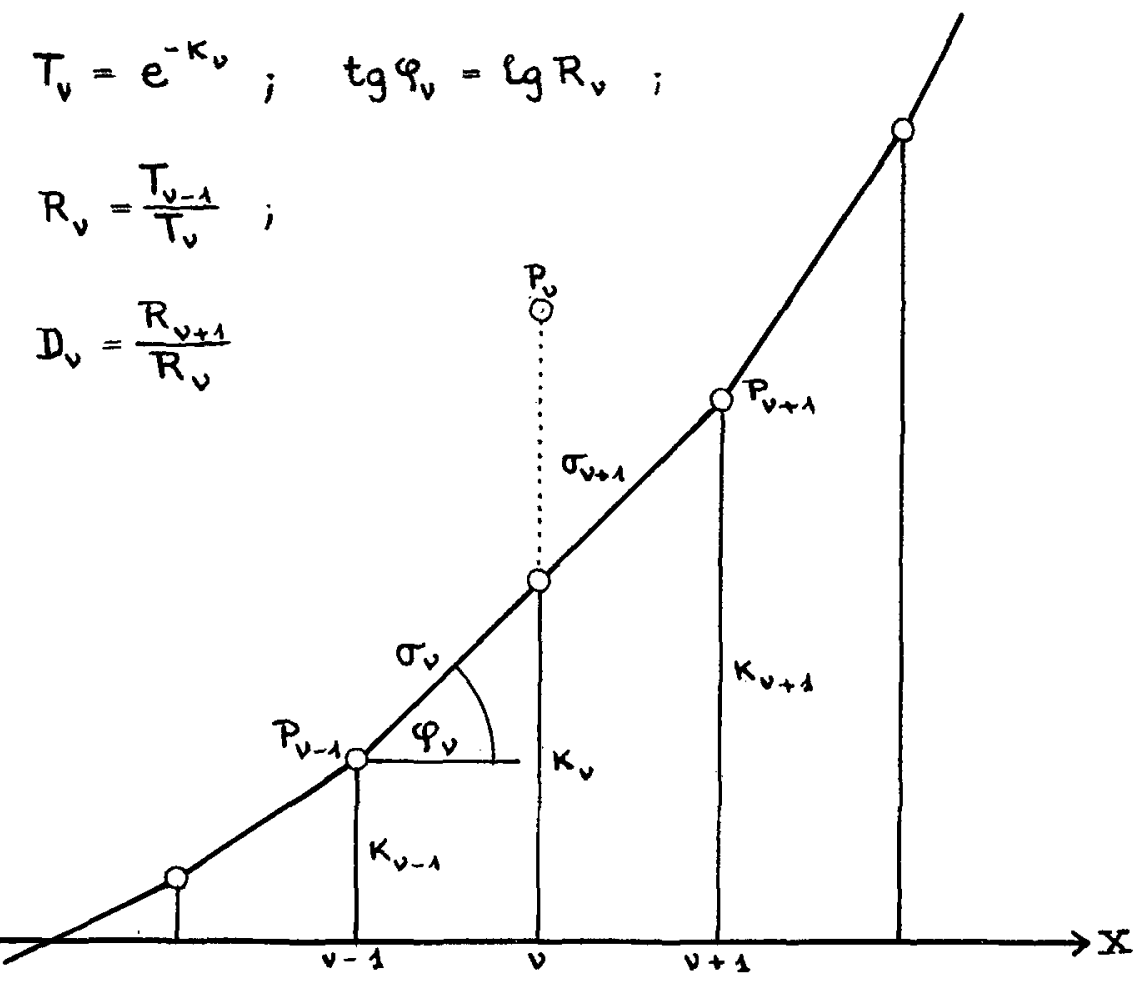

Fig. 1.

Il résulte $\mathrm{du}$ caractère convexe de $D$ que l'on a $x_{v+1}-x_{v} \geqq x_{v}-x_{v-1}$, donc $(10,4)$

$$
R_{v+1} \geqq R_{v}, D_{v} \geqq \mathrm{r}
$$

Si $D_{v}>\mathrm{I}, \nu$ sera appelé un indice principal de $D$. Nous comptons en outre $n_{0}$ aux indices principaux de $D$, si $n_{0}>-\infty$, et $n^{\prime}$, si $n^{\prime}<\infty$. Les indices principaux correspondent aux sommets du diagramme $D$. Donc pour un indice principal $v$ on a

$$
\left|a_{v}\right|=T_{v}>0
$$

Si $n^{\prime}=n_{0}$, tous les $T_{v}$ sont o sauf $T_{n_{0}}=\left|a_{n_{0}}\right|$, et $n_{0}$ est l'indice principal. Si tous les $a_{v}$ sont 0 , on fait chaque $T_{v}=0$.

Il résulte de $(10,2)$ $(10,5)$

$$
\frac{T_{n+v}}{T_{n}}=\frac{\mathrm{I}}{R_{n+1} \ldots R_{n+v}}, \quad\left(n_{0} \leqq n<n+v \leqq n^{\prime}\right)
$$

16-39615. Acta mathematica. 72. Imprimé le 2 mars 1940. 
I I. En vertu de $($ IO, 4$)$ l'expression

$$
\mathfrak{M}_{f}=\sum_{v=-\infty}^{\infty} T_{v} z^{n}
$$

est une série normale telle que, si $F(z)=\sum_{v=-\infty}^{+\infty} t_{v} z^{v}, t_{v} \geqq 0$ est une série normale majorante de $f(z)$, on a $t_{v} \geqq T_{v}$. Nous appellerons (I I, I) la majorante newtonienne de $f(z)$. Elle coïncide évidemment avec la majorante newtonienne, définie dans les Nos. 2, 3, si $f(z)$ est un polynôme.

Il résulte de (Io,4) que, si $f(z)$ est une série de Taylor ne se réduisant pas à un polynome, il existe un $\varrho$ tel que ${ }^{1}$

$$
R_{\nu} \uparrow \varrho ; \frac{T_{v-1}}{T_{\nu}} \rightarrow \varrho ; \sqrt[\nu]{T_{\nu}} \rightarrow \frac{\mathbf{I}}{\varrho} .
$$

$\varrho$ est donc le rayon de convergence de $\mathfrak{M}_{f}$ et, si $\varrho^{\prime}$ est celui de $f(z)$, on a $\varrho^{\prime} \geqq \varrho$. Or, si un nombre infini de points représentatifs $P_{v}$ appartiennent à $D$, on a pour une suite partielle des indices $v_{x}:\left|a_{v_{x}}\right|=T_{v_{x}}$, donc par (I I, 2)

done $\varrho^{\prime}=\varrho$.

$$
\sqrt[v_{x}]{\left|a_{v_{x}}\right|} \rightarrow \frac{I}{\varrho}, \varrho^{\prime} \leqq \varrho,
$$

Dans le cas où $D$ ne contient qu'un nombre fini des points $P_{\nu}, D$ contient une demi-droite

Donc pour $\nu \geqq \nu_{0}$

$$
\text { (L) } \quad y=\alpha x+\beta, x \geqq v_{0} \text {. }
$$

$$
T_{\nu}=\left(e^{-\alpha}\right)^{v} e^{-\beta}=\frac{\mathrm{I}}{e^{\beta} R^{v}}
$$

en posant $R=e^{\alpha}$. Il en résulte pour $\nu \geqq \nu_{0}, R_{v}=\frac{T_{\nu-1}}{T_{v}}=R$, donc $R=\varrho$. Mais d'un autre côté, d'après la propriété $3^{\circ}$ ) du No. 7, pour une série partielle des indices $\nu_{x}$ les angles des rayons vecteurs correspondant aux points représentatifs $P_{v_{x}}$ avec la demi-droite $L$ convergent vers $o$. Il en résulte

$$
-\frac{\lg \left|a_{v_{x}}\right|}{v_{x}} \rightarrow \lg \varrho
$$

\footnotetext{
1 Les symboles $\uparrow, \downarrow$ sont a lire: .. tend en croissant resp. décroissant vers ...
} 


$$
\sqrt[v_{x}]{\left|a_{v_{x}}\right|} \rightarrow \frac{1}{\varrho}
$$

Donc dans ce cas aussi $\varrho^{\prime} \leqq \varrho$, donc $\varrho^{\prime}=\varrho$.

Donc, dans le cas d'une série de Taylor le rayon de convergence de $\mathfrak{M}_{f}$ est égal à celui de $f(z)$.

I2. Supposons maintenant que $f(z)$ soit une série de Laurent et que $D$ possède plus d'un côté, ou bien que, si $D$ se réduit à une droite, il y a au moins un point représentatif sur cette droite qu'on peut supposer être $P_{0}$. Alors on voit comme au No. I I, que les deux séries

$$
\sum_{\nu=0}^{\infty} a_{\nu} z^{\nu} \text { et } \sum_{\nu=0}^{\infty} T_{\nu} z^{v}
$$

ont le même rayon de convergence. Et il en est évidemment de mêrne pour les séries

$$
\sum_{\nu=0}^{\infty} a_{-v} z^{\nu} \text { et } \sum_{\nu=0}^{\infty} T_{-v} z^{v}
$$

Donc, dans ce cas $f(z)$ possède la même couronne de convergence que $\mathfrak{M}_{f}$.

Si enfin $D$ se réduit à une droite

$$
\text { (L) } \quad y=\alpha \nu+\beta
$$

qui ne contient aucun $P_{v}$, la condition $5^{\circ}$ ) du No. 7 est satisfaite et l'on pourra appliquer aux deux séries $\sum_{\nu=-\infty}^{0} a_{\nu} z^{\nu}, \sum_{\nu=0}^{\infty} a_{\nu} z^{\nu}$ les mêmes considérations que dans le cas précédent. Donc, puisque maintenant $R_{v}=R(-\infty<y<\infty)$, le rayon de convergence de la série

$$
\sum_{v=0}^{\infty} a_{v} z^{v}
$$

est au plus égal à $R$ et d'après $(\mathrm{I} \mathrm{I}, 2) \geqq R$, donc il est égal à $R$. De même, le rayon de convergence de la série

$$
\sum_{\nu=0}^{\infty} a-v z^{\nu}
$$


est égal à $\frac{I}{R}$. Donc dans ce cas la série $f(z)$ ne peut être convergente que pour $|z|=R$. Sa majorante newtonienne diverge partout. Donc:

V. La majorante newtonienne $\mathfrak{M}_{f}$ de $f(z)$ possède la mème couronne de convergence que $f(z)$. Si cette couronne de convergence est $\varrho_{1}<|z|<\varrho$ on a

$$
R_{v} \uparrow \varrho \quad(\nu \rightarrow \infty) ; R_{v} \downarrow \varrho_{1} \quad(v \rightarrow-\infty),
$$

si la suite correspondante de $R_{v}$ existe.

Soit $A(z)=\sum_{\nu=m}^{n} a_{v} z^{\nu}$ un segment» de $f(z)$, tel que $m, n$ soient des indices principaux ou plus généralement, que les points réprésentatifs $P_{m}, P_{n}$ soient situés sur le diagramme de $f(z)$. Alors il résulte de la construction de $\mathfrak{M}(z)$ que la majorante newtonienne de $A(z)$ est le segment $\sum_{\nu=m}^{n} T_{\nu} z^{v}$ de $\mathfrak{M}_{f}$.

Dans les considérations de ce paragraphe nous avons utilisé pour la construction du diagramme $D$ les logarithmes naturels. Il va sans dire que l'on peut aussi employer les logarithmes décimals et c'est ce qu'on va faire généralement dans les applications. Le diagramme $D$ restera essentiellement le même - c'est la question de l'échelle utilisée sur l'axe des $y$. La grandeur $x_{v}$ sera différente. Mais, si l'on remplace dans les formules (3, I), (IO, I) et (IO, 2) les puissances de $e$ par les puissances de ro, les nombres $R_{v}, T_{\nu}, D_{v}$ resteront les mêmes, ainsi que les indices principaux.

\section{$\S 4$. Transformations du diagramme et relations caractéristiques.}

I3. Si l'on multiplie $f(z)$ par une constante $a \neq 0$, le diagramme $D$ subit un déplacement parallèle à la direction négative de l'axe des $y$ de longueur $\lg |a|$.

La majorante newtonienne de $f(z)$ se multiplie par $|a|$. -

Si l'on remplace $z$ par $\frac{1}{z}$, les points représentatifs $P_{v}$ sont remplacés par leurs symétriques par rapport à l'axe des $y$.

La majorante newtonienne de $f\left(\frac{\mathrm{I}}{z}\right)$ s'obtient de $\mathfrak{M}_{\mathrm{g}}$ en y remplaçant $z$ par $\frac{\mathrm{I}}{z}$.

Si l'on multiplie $f(z)$ par $z^{m}, D$ subit un déplacement parallèle à l'axe des $x$ de longueur $m$. La majorante newtonienne de $z^{m} f(z)$ est égal à $z^{m} \mathfrak{M}_{f}$.

$\mathrm{Si}$, en particulier, $f(z)$ est un polynome du degré exact $n$ et $f(0) \neq 0$, on 
obtient la suite des inclinaisons numériques du diagramme de $f_{1}(z)=z^{n} f\left(\frac{I}{z}\right)$ en prenant les inverses des inclinaisons numériques $R_{v}$ du diagramme de $f(z)$ et en les écrivant dans l'ordre inverse.

14. Si l'on remplace $z$ dans $f(z)$ par $\alpha z,|\alpha|=e^{\sigma}$, les nouveaux points représentatifs $P_{v}$ auront les cordonnées

$$
\left(\nu, \lg \frac{\mathbf{I}}{\left|a_{v}\right|}-v \sigma\right)
$$

donc, on les déduit des points représentatifs de $f(z)$ par la transformation

$$
\xi=x, \quad \eta=y-\sigma x,
$$

par laquelle, généralement, la droite $y=m x+A$ devient $\eta=(m-\sigma) \xi+A$. C'est une affinité qui appartient aux déformations homogènes élémentaires considérées dans la théorie de l'élasticité. $\sigma$ est alors le glissement correspondant.

Donc, si l'on passe de $f(z)$ à $f(\alpha z), D$ subit la transformation $(14,2)$ qui ne change pas les indices principaux. Les inclinaisons numériques sont divisées par $|\alpha|$. Les déviations restent les mêmes.

Pour $\sigma=m$ la droite $y=m x+A$ devient parallèle à l'axe des $x$. Donc, en choisissant $\alpha$ convenablement on peut faire que le segment élémentaire $\sigma_{\nu}$ de $D$ devienne parallèle à l'axe des $x$.

Donc, en choisissant $a \neq 0, \alpha \neq 0$ on peut faire que $D$ pour $a f(\alpha z)$ contienne l'intervalle $\langle v-\mathrm{I}, v\rangle$ de l'axe des $x(v$ entier), si cet intervalle est contenu dans la projection du diagramme de $f(z)$ sur l'axe des $x$.

La majorante newtonienne de af $(\alpha z)$ est évidemment égale à $|a| \mathfrak{M}_{f}(|\alpha| z)$.

I5. Soient maintenant $n_{\mu-1}, n_{\mu}$ deux indices principaux consécutifs de $D$, on a évidemment

$$
\left|a_{n_{\mu}}\right|=T_{n_{\mu}},\left|a_{n_{\mu-1}}\right|=T_{n_{\mu-1}} .
$$

Donc, puisque

$$
R_{n_{\mu-1}+1}=R_{n_{\mu-1}+2}=\cdots=R_{n_{\mu}}
$$

on a:

$$
R_{n_{\mu}}=\left(\frac{T_{n_{\mu-1}}}{T_{n_{\mu}}}\right)^{\frac{1}{n_{\mu}-n_{\mu-1}}}=\left|\frac{a_{n_{\mu-1}}}{a_{n_{\mu}}}\right|^{\frac{1}{{ }_{\mu}-n_{\mu-1}}},
$$




$$
\left|\frac{a_{n_{\mu-1}}}{a_{n_{\mu}}}\right|=R_{n_{\mu}}^{n_{\mu}-n_{\mu-1}}
$$

Et, en écrivant que pour $n_{\mu-1}<\nu<n_{\mu}$ le point $P_{v}$ est situé au dessus du segment élémentaire $\sigma_{v}$ de $D$, ou $\operatorname{sur} \sigma_{v}$,

$$
\left|\frac{a_{\nu}}{a_{n_{\mu-1}}}\right| \leqq R_{n_{\mu}}^{n_{\mu-1}-\nu},\left|\frac{a_{n_{\mu}}}{a_{\nu}}\right| \geqq R_{n_{\mu}}^{v-n_{\mu}}, \quad n_{\mu-1} \leqq \nu \leqq n_{\mu} .
$$

D'autre part, exprimons que pour $v>n_{\mu}$ le point $P_{v}$ est situé au dessus du prolongement du segment élémentaire $\sigma_{n_{\mu}}$ à droite, on obtient:

$$
\left|\frac{a_{v}}{a_{n_{\mu}}}\right|<R_{n_{\mu}}^{n_{\mu}-v},\left|\frac{a_{v}}{a_{n_{\mu-1}}}\right|<R_{n_{\mu}}^{n_{\mu-1}-v} .
$$

Et d'un autre côté, en exprimant que pour $v<n_{\mu-1}$ le point $P_{v}$ est situé au dessus du prolongement $\mathrm{du}$ segment élémentaire $\sigma_{n_{\mu}} \grave{a}$ gauche, on obtient les mêmes inégalités. Donc

$$
\left|\frac{a_{v}}{a_{n_{\mu}}}\right|<R_{n_{\mu}}^{n_{\mu}-v}, \quad\left|\frac{a_{v}}{a_{n_{\mu-1}}}\right|<R_{n_{\mu}}^{n_{\mu-1-v}}, \quad\left(\nu<n_{\mu-1}, \nu>n_{\mu}\right) .
$$

On a donc en particulier pour tout $\nu$ et tout $\mu$

$$
\left|a_{v}\right| R_{n_{\mu}}^{\nu} \leqq\left|a_{n_{\mu-1}}\right| R_{n_{\mu}}^{n_{\mu-1}},\left|a_{\nu}\right| R_{n_{\mu}}^{v} \leqq\left|a_{n_{\mu}}\right| R_{n_{\mu}}^{n_{\mu}}
$$

Les relations $\left(I_{5}, 3\right),\left(I_{5}, 4\right),\left(I_{5}, 5\right)$ sont évidemment nécessaỉres et suffisantes pour que les indices $n_{\mu-1}, n_{\mu}$ soient deux indices principaux consécutifs.

De l'autre côté, on a pour les $T_{v}, n_{\mu-1} \leqq \nu \leqq n_{\mu}$, puisque les points $\left(\nu, \lg T_{v}\right)$ sont situés sur une droite:

$$
T_{\nu}=T_{n_{\mu-1}}^{\frac{n_{\mu}-v}{n_{\mu}-1}} T_{n_{\mu}}^{\frac{\nu-n_{\mu-1}}{n_{\mu}-n_{\mu-1}}}, \quad n_{\mu-1} \leqq \nu \leqq n_{\mu} .
$$

Enfin, pour trois indices $n^{\prime}, n, n^{\prime \prime}$ avec $n^{\prime}<n<n^{\prime \prime}$, si les $T_{v}$ correspondants ne disparaissent pas, le point $\left(n,-\lg T_{n}\right)$ est situé au dessous ou sur le segment de droite joignant les points $\left(n^{\prime},-\lg T_{n^{\prime}}\right)$ et $\left(n^{\prime \prime},-\lg T_{n^{\prime \prime}}\right)$. Il en résulte

$$
T_{n} \geqq T_{n^{\prime}}^{\frac{n^{\prime \prime}-n}{n^{\prime \prime}-n^{\prime}}} \frac{n-n^{\prime}}{T_{n^{\prime \prime}}^{n^{\prime \prime}-n^{\prime}}}, \quad n^{\prime}<n<n^{\prime \prime}, \quad T_{n^{\prime}} T_{n^{\prime \prime}} \neq \mathrm{o}
$$

formule valable pour toute série normale. 
Nous allons enfin déduire quelques conditions analytiques pour les indices principaux et une détérmination directe des inclinaisons numériques. À cet effet désignons par $L_{n}^{+}$la demi-droite issue du point représentatif $P_{n}, n_{0}<n<n^{\prime}$, allant de gauche à droite et telle que tous les points $P_{v}, v>n$ soient situés ou bien $\operatorname{sur} L_{n}^{+}$ou bien au dessus et que l'angle $\vartheta_{+}$formé par cette demi-droite avec l'axe positif des $x$ soit maximum. On a

$$
\operatorname{tg} \vartheta_{+}=\operatorname{Min}_{\nu>0} \frac{\lg \left|a_{n}\right|-\lg \left|a_{n+v}\right|}{\nu}
$$

D'un autre côté soit $L_{n}^{-}$une demi-droite venant de gauche et finissant dans le point $P_{n}$ et telle que tous les points $P_{\nu}, \nu<n$ soient situés ou bien sur $L_{n}^{-}$ou bien au dessus et que l'angle $\vartheta_{-}$de la direction de $L_{n}^{--}$avec la direction de l'axe positif des $x$ soit minimum. On a

$$
\operatorname{tg} \vartheta_{-}=\operatorname{Max}_{x \rightarrow 0} \frac{\lg \left|a_{n-x}\right|-\lg \left|a_{n}\right|}{x}
$$

Or, si $n$ est un indice principal, les directions de $L_{n}^{-}$et $L_{n}^{+}$sont celles de deux côtés du diagramme de Newton, aboutissant à $P_{n}$. On a donc alors

$$
\lg R_{n}=\operatorname{tg} \vartheta_{-}<\lg R_{n+1}=\operatorname{tg} \vartheta_{+} .
$$

Si $P_{n}$ est situé sur un côté du diagramme de Newton sans être un sommet de ce diagramme on a évidemment

$$
\lg R_{n}=\operatorname{tg} \vartheta_{-}=\operatorname{tg} \vartheta_{+}=\lg R_{n+1}
$$

Enfin, si $P_{n}$ est situé au dessus du côté correspondant du diagramme on a

$$
\left|a_{n}\right|<T_{n}
$$

et la relation précédente est satisfaite si l'on y remplace $\left|a_{n}\right|$ par $T_{n}$. Il en résulte que

a) La condition nécéssaire et suffisante pour que $n, n_{0}<n<n^{\prime}$, soit un indice principal du diagramme est

$$
\frac{\operatorname{Min}_{\nu>0}\left|\frac{a_{n}}{a_{n+\nu}}\right|^{\frac{1}{v}}}{\operatorname{Max}_{x>0}\left|\frac{a_{n-x}}{a_{n}}\right|^{\frac{1}{x}}}>\mathrm{I} .
$$


La condition nécessaire et suffisante pour $\left|a_{m}\right|=T_{n}$ sans que n soit un indice principal est

$$
\frac{\operatorname{Min}_{\nu>0}\left|\frac{a_{n}}{a_{n+v}}\right|^{\frac{1}{v}}}{\operatorname{Max}_{x>0}\left|\frac{a_{n-x}}{a_{n}}\right|^{\frac{1}{x}}}=1 .
$$

Si $(15,8)$ ou $\left(15,8^{\circ}\right)$ est satisfaite, on a

$$
R_{n}=\operatorname{Max}_{x>0}\left|\frac{a_{n-x}}{a_{n}}\right|^{\frac{1}{x}}, \quad R_{n+1}=\operatorname{Min}_{\nu>0}\left|\frac{a_{n}}{a_{n+\nu}}\right|^{\frac{1}{v}} .
$$

b) Si l'on a

$$
\frac{\operatorname{Min}_{v>0}\left|\frac{a_{n}}{a_{n+v}}\right|^{\frac{1}{v}}}{\operatorname{Max}_{x>0}\left|\frac{a_{n-x}}{a_{n}}\right|^{\frac{1}{x}}}<\mathrm{I},
$$

on déterminera la racine positive $x_{0}$ de l'équation

$$
\frac{\operatorname{Min}_{v>0}\left(\frac{x}{\left|a_{n+v}\right|}\right)^{\frac{1}{v}}}{\operatorname{Max}_{x>0}\left(\frac{\left|a_{n-x}\right|}{x}\right)^{\frac{1}{x}}}=\mathrm{I} .
$$

Alors on aura

$\left(\right.$ I $\left.5,9^{\circ \circ}\right) \quad T_{n}=x_{0}, R_{n}=R_{n+1}=\operatorname{Min}_{\nu>0}\left(\frac{x_{0}}{\left|a_{n+v}\right|}\right)^{\frac{1}{v}}=\operatorname{Max}_{x>0}\left(\frac{\left|a_{n-x}\right|}{x_{0}}\right)^{\frac{1}{x}}$.

Si $f(z)$ est un polynôme, il est plus simple de déterminer les indices principaux et les inclinaisons numériques successivement. Nous allons expliquer ce procédé de calcul sur un exemple.

Soit

$$
\begin{aligned}
f(z) \equiv 6 z^{10}+60 z^{9}+200 z^{8}+600 z^{7}+1200 z^{6}+ \\
+100 z^{5}+300 z^{4}+1800 z^{3}+1800 z^{2}+600 z+600 .
\end{aligned}
$$

On a ici $\mathrm{I}=\frac{a_{1}}{a_{0}}<\left(\frac{a_{2}}{a_{0}}\right)^{\frac{1}{2}}=\sqrt{3} ;$ donc $R_{1}=R_{2}$. De l'autre côté on a évidemment 


$$
\sqrt{3}=\left(\frac{a_{2}}{a_{0}}\right)^{\frac{1}{2}}>\frac{a_{3}}{a_{2}},\left(\frac{a_{4}}{a_{2}}\right)^{\frac{1}{2}},\left(\frac{a_{5}}{a_{2}}\right)^{\frac{1}{3}}, \ldots,\left(\frac{a_{10}}{a_{2}}\right)^{\frac{1}{8}}
$$

Donc 2 est le premier indice principal après o et l'on a $R_{1}=R_{\mathrm{a}}=\frac{\mathrm{I}}{\sqrt{3}} \cdot$ En partant de $a_{\mathrm{g}}$ on a

$$
\mathrm{I}=\frac{a_{3}}{a_{2}}>\frac{a_{4}}{a_{3}},\left(\frac{a_{5}}{a_{3}}\right)^{\frac{1}{2}}, \ldots,\left(\frac{a_{10}}{a_{3}}\right)^{\frac{1}{7}}
$$

donc 3 est un indice principal et l'on a $R_{3}=$ I.

En partant de $a_{3}$ on obtient

$$
\frac{a_{4}}{a_{3}}<\left(\frac{a_{6}}{a_{3}}\right)^{\frac{1}{3}},\left(\frac{a_{5}}{a_{3}}\right)^{\frac{1}{2}}<\left(\frac{a_{6}}{a_{3}}\right)^{\frac{1}{3}}
$$

Donc $R_{4}=R_{5}=R_{6}$. De l'autre côté on a

$$
\sqrt[3]{\frac{2}{3}}=\left(\frac{a_{6}}{a_{3}}\right)^{\frac{1}{3}}>\frac{a_{7}}{a_{6}},\left(\frac{a_{8}}{a_{6}}\right)^{\frac{1}{2}},\left(\frac{a_{9}}{a_{6}}\right)^{\frac{1}{3}},\left(\frac{a_{10}}{a_{6}}\right)^{\frac{1}{4}}
$$

Done 6 est le $4^{\text {ème }}$ indice principal et l'on a $R_{4}=R_{5}=R_{6}=\sqrt[3]{\frac{3}{2}}$. En partant de $a_{6}$ on obtient

$$
\frac{a_{7}}{a_{6}}=\frac{\mathrm{I}}{2}>\frac{a_{8}}{a_{7}}=\frac{\mathrm{I}}{3}>\frac{a_{9}}{a_{8}}=\frac{3}{\mathrm{IO}}>\frac{a_{10}}{a_{9}}=\frac{\mathrm{I}}{\mathrm{IO}} .
$$

Donc les indices $7,8,9$, 10 sont des indices principaux et l'on a

$$
R_{7}=2, R_{8}=3, R_{9}=\frac{\text { IO }}{3}, R_{10}=\text { IO }
$$

I6. VI. Supposons que pour deux séries de Laurent

$$
f(z)=\sum_{\nu=-\infty}^{\infty} a_{\nu} z^{v}, \quad G(z)=\sum_{\nu=-\infty}^{\infty} b_{\nu} z^{\nu}
$$

la relation suivante soit satisfaite:

$$
f(z)-G(z) \ll \delta \mathfrak{M}_{G}
$$$$
(0<\delta<1)
$$

Alors on $a$

$$
(\mathrm{I}-\delta) \mathfrak{M}_{G} \ll \mathfrak{M}_{f} \ll(\mathrm{I}+\delta) \mathfrak{M}_{G}
$$

et, en désignant par $R_{v}^{*}, D_{v}^{*} ; R_{v}, D_{v}$ resp. les inclinaisons numériques et les déviations de $G(z)$ et $f(z)$,

17-39615. Acta mathematica. 72. Imprimé le 2 mars 1940. 


$$
\begin{gathered}
\frac{\mathrm{I}-\delta}{\mathrm{I}+\bar{\delta}} \leqq \frac{R_{v}^{*}}{R_{v}} \leqq \frac{\mathrm{I}+\delta}{\mathrm{I}-\delta}, \\
\left(\frac{\mathrm{I}-\delta}{\mathrm{I}+\delta}\right)^{2} \leqq \frac{D_{v}^{*}}{D_{v}} \leqq\left(\frac{\mathrm{I}+\delta}{\mathrm{I}-\delta}\right)^{2},
\end{gathered}
$$

autant que les inclinaisons numériques $R_{v}$ et les déviations $D_{v}$ existent pour l'indice $\nu$.

Démonstration. Posons

$$
\mathfrak{M}_{f}=\sum_{\nu=-\infty}^{\infty} T_{\boldsymbol{v}} z^{v}, \quad \mathfrak{M}_{G}=\sum_{v=-\infty}^{\infty} \beta_{\boldsymbol{v}} z^{v},
$$

alors il résulte de $(I 6,2)$

$$
\left|a_{v}\right| \leqq\left|b_{v}\right|+\delta \beta_{v} \leqq(\mathrm{I}+\delta) \beta_{\nu},
$$

c'est-à-dire que $f(z)$ est majorée par la série $(\mathrm{I}+\delta) \mathfrak{M}_{G}$ qui est évidemment normale avec $\mathfrak{M}_{G}$. Donc

$$
\mathfrak{M}_{f} \ll(\mathrm{I}+\delta) \mathfrak{M}_{G} .
$$

Soit maintenant $n$ un indice principal de $G(z)$, alors on a

$$
T_{n} \geqq\left|a_{n}\right| \geqq\left|b_{n}\right|-\delta \beta_{n}=(\mathrm{r}-\delta) \beta_{n} ;
$$

or, je dis que l'inégalité

$(16,6)$

$$
T_{n} \geqq(\mathbf{1}-\delta) \beta_{n}
$$

est valable non seulement pour les indices principaux de $G(z)$ mais pour chaque indice $n$. En effet, soit $n$ un indice situé entre deux indices principaux consécutifs $n^{\prime}, n^{\prime \prime}$ de $G(z)$ :

$$
n^{\prime}<n<n^{\prime \prime}
$$

Alors d'après $\left(I_{5}, 6\right)$ appliquée aux $\beta_{y}$ :

$$
\beta_{n}=\beta_{n^{\prime}}^{\frac{n^{\prime \prime}-n}{n^{\prime \prime}-n^{\prime}}} \beta_{n^{\prime \prime}}^{\frac{n-n^{\prime}}{n^{\prime \prime}-n^{\prime}}}
$$

donc, comme $(16,6)$ est déjà démontrée pour les indices principaux,

$$
(\mathrm{I}-\bar{\delta}) \beta_{n} \leqq T_{n^{\prime}}^{\frac{n^{\prime \prime}-n^{\prime}}{n^{\prime \prime}}} T_{n^{\prime \prime}}^{\frac{n-n^{\prime}}{n^{\prime \prime}}}
$$

et l'on obtient (I6.6) en utilisant $(15,7)$. 
De la relation $(16,3)$ ainsi démontrée résulte la formule

$$
(\mathrm{I}-\delta) \beta_{n} \leqq T_{n} \leqq(\mathrm{I}+\delta) \beta_{n}
$$

dont on obtient $(16,4)$ et $(16,5)$ par division.

On peut tirer du théorème VI un énoncé que nous aurons à utiliser plus bas.

Considérons une expression »finie»

$$
f(z)=\sum_{v=l_{1}}^{l_{2}} a_{v} z^{v}, \quad l_{2}-l_{1}=n-\mathbf{I}
$$

Les $n$ modules $\left|a_{v}\right|, v=l_{1}, l_{1}+\mathrm{I}, \ldots, l_{2}$ peuvent être considéré comme coordonnées d'un point $P$ de l'espace $R_{n}$ à $n$ dimensions et les nombres $T_{v}, R_{v}, D_{v}$ correspondant à $f(z)$ sont alors des fonctions du point $P$.

Alors chaque $T_{v}$ est une fonction continue dans tous les points $P_{0}$ de $R_{n}$.

En effet, si $P_{0}$ est à l'origine de $R_{n}$, tous les $T_{v}$ y disparaissent. Pour un point $P$ suffisamment approché à $P_{0}$ les ordonnées de tous les points du diagramme de Newton correspondant sont arbitrairement grands et les $T_{v}$ correspondants arbitrairement petits.

Si $P_{0}$ n'est pas à l'origine de $R_{n}$, soient $n_{0}$ le premier et $n^{\prime}$ le dernier indice pour lesquels les coordonnées de $P$ sont différentes de o. Alors pour les indices $\nu$ avec $n_{0} \leqq \nu \leqq n^{\prime}$ la continuité de $T_{\nu}$ est une conséquence immédiate du théorème VI. Quant aux indices $v<n_{0}$ ou $>n^{\prime}$, les $T_{v}$ correspondant à $P_{0}$ sont o. Mais alors pour les points $P$ de $R_{n}$ suffisamment approchés de $P_{0}$, les $T_{v}$ correspondants sont arbitrairement petits, puisque les points correspondants du diagramme de Newton possèdent des ordonnées arbitrairement grandes.

Il en résulte en particulier que chaque $R_{v}$ et $D_{v}$ est continu dans tous les points de $R_{\imath}$ où cette expression possède une valeur déterminée. (On considérera naturellement $\infty$ aussi comme une valeur déterminée et la continuité est dans ce cas-ci à définir comme la continuité de l'expression inverse.)

Pour le cas d'une expression $(6, \mathrm{I})$ infinie le fait correspondant doit être énoncé d'une façon plus restreinte. Considérons une suite infinie des expressions $f_{\mu}(z)=\sum_{\nu=-\infty}^{\infty} a_{v}^{(\mu)} z^{\nu}$ et l'expression $f(z)=\sum_{v=-\infty}^{\infty} a_{\nu} z^{\nu}$. Soient $\sum_{\nu=-\infty}^{\infty} T_{v}^{(\mu)} z^{\nu}, \sum_{\nu=-\infty}^{\infty} T_{v} z^{v}$ resp. les majorantes newtoniennes de $f_{\mu}(z), f(z)$. Supposons que pour chaque $\nu$ on a $a_{v}^{(\mu)} \rightarrow a_{v}$ avec $\mu \uparrow \infty$. Supposons en plus que deux entiers $l_{1}, l_{2}, l_{1}<l_{2}$ possèdent la propriété que pour chacune des séries $f_{\mu}(z), f(z)$ les points représen- 
tatifs aux indices $l_{1}, l_{2}$ soient situés sur le diagramme de la série correspondante. ${ }^{1}$ Il en résulte évidemment d'après No. I 2 que les majorantes newtoniennes des expressions $\sum_{v=l_{1}}^{l_{2}} a_{v}^{(\mu)} z^{v}$ convergent avec $\mu \uparrow \infty$ vers la majorante newtonienne de $\sum_{\nu-l_{1}}^{l_{2}} a_{\nu} z^{v}$, donc, on a pour chaque $\nu, l_{1} \leqq \nu \leqq l_{2}$, avec $\mu \uparrow \infty: T_{\nu}^{(\mu)} \rightarrow T_{v}$.

Il en résulte en particulier, en désignant resp. par $R_{v}^{(\mu)}, D_{v}^{(\mu)}, R_{v}, D_{v}$ les inclinaisons numériques et les déviations de $f_{\mu}(z)$ et $f(z)$ que l'on a $R_{v}^{\langle(\mu)} \rightarrow R_{v}$ avec $\mu \uparrow \infty$, si $l_{1}<v \leqq l_{2}$ et $R_{v}$ possède une valeur déterminée, et $D_{v}^{(\mu)} \rightarrow I_{v}$, si $l_{1}<\nu<l_{2}$ et $D_{1}$ possède une valeur déterminée. ${ }^{2}$

Le résultat démontré est évidemment applicable à une expression

$$
f(z ; P)=\sum_{\nu=-\infty}^{\infty} a_{v}(P) z^{v}
$$

où les coefícients $a_{v}(P)$ sont des fonctions continues du point parametral $P$ variant dans un ensemble $\mathfrak{M}$, pour les indices $y$ satisfaisant uniformément en $P$ aux conditions indiquées. Alors les coefficients de la majorante newtonienne de $f(z ; P)$ et les inclinaisons numériques et les déviations correspondantes sont fonctions continues de $P$ sur $\mathfrak{D}$.

\section{CHAPITRE II. Les bornes des racines dépendant des inclinaisons numériques du diagramme de Newton.}

\section{$\S$ 5. Premier théorème fondamental.}

I 7. VII. Premier théorème fondamental. Soit

$$
f(z)=\sum_{v=0}^{\infty} a_{v} z^{v}, \quad a_{0} \neq 0
$$

1 Il est d'ailleurs superflu de faire cette hypothèse sur $f(z)$ explicitement. En effet, il résulte immédiatement des conditions $(15,8)$ et $\left(15,8^{\circ}\right)$, que si un indice $l$ jouit pour une suite infinie des expressions $f_{\mu l}(z)$ de la proprité que les points représentatifs d'indice $l$ pour.ces séries sont situés sur leurs diagrammes correspondants, il en est de même pour l'expression limite $f(z)$.

${ }^{2}$ On peut généraliser nos conditions pour $v$ de la façon suivante: pour $T_{v}^{(\mu)} \rightarrow T_{v}$ il suffit qu'il existe deux nombres fixes $k_{1}, k_{2}$ et pour chaque $\mu$ deux entiers $l_{1}^{\prime}(\mu), l_{2}(\mu)$ tels que l'on ait $k_{1} \leqq l(\mu) \leqq \nu \leqslant l(\mu) \leqslant k_{2}$. On obtient une condition suffisante pour $\boldsymbol{R}_{i}^{(u)} \rightarrow R_{\nu}$ en remplaçant ici

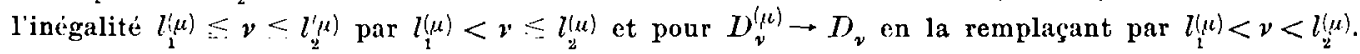
Notons enfin que l'on a sans aucume condition supplémentaire pour $\nu: T_{\nu} \leq \lim _{--} T_{v}^{(\mu)}$. 
une série entièse (pouvant se réduive à un polymòme) sans zéro à l'origine, à rayon de convergence positif. Soient
$\left(17,1^{\circ}\right)$
$\zeta_{1},\left|\zeta_{1}\right| \leqq\left|\zeta_{2}\right| \leqq \cdots$

les zéros de $f(z)$ dans le cercle de convergence, rangés par ordre des modules croissants et $R_{y}$ les inclinaisons numériques du diagramme de Neuton de $f(z)$.

A. Si $f(z)$ possède pour un entier $p \geqq \mathrm{I}$ dans le cercle de convergence au moins $p$ zéros, on a

$$
\begin{gathered}
\frac{\left|\zeta_{p}\right|}{R_{p}} \geqq \mathrm{I}-\left(\begin{array}{l}
\mathrm{I} \\
2
\end{array}\right)^{\frac{1}{p}}, \\
\prod_{x=1}^{p}\left(\mathrm{I}-\frac{\left|\zeta_{x}\right|}{R_{p}}\right) \leqq \frac{\mathrm{I}}{2} \text { si }\left|\zeta_{p}\right| \leqq R_{1} .
\end{gathered}
$$

B. La condition nécessaive et suffisante pou que l'on ait pour $\left|\zeta_{p}\right|<R_{p}$ :

$(17,4)$

$$
\prod_{x \rightarrow 1}^{p}\left(\mathrm{I}-\begin{array}{c}
\left|\zeta_{x}\right| \\
R_{p}
\end{array}\right)=\stackrel{\mathrm{I}}{2}
$$

est, que $f(z)$ puisse ôtre mise sous la forme

$$
f(z)=A \psi\left(\begin{array}{c}
z \\
R_{p} \varepsilon
\end{array}\right)
$$

oir $A \neq \mathrm{o},|\varepsilon|=\mathrm{I}_{\mathrm{I}}$ et pour $p$ nombres $\varrho_{v}$ arec $\mathrm{o}<\varrho_{1} \leqq \cdots \leqq \varrho_{p}<\mathrm{I}, \prod_{x=1}^{p}\left(\mathrm{I}-\varrho_{x}\right)=\frac{\mathrm{I}}{2}$ :

$$
\psi(z)=2^{\prod_{x-1}^{n}\left(z-\varrho_{x}\right)}
$$

La condition nécessaive et suffisante pou que l'on ait

$$
\left|\zeta_{p}\right|=-\left(\mathrm{I}-\left(\begin{array}{l}
\mathrm{I} \\
2
\end{array}\right)^{p}\right) R_{p}
$$

est, que $f(z)$ soit égale d̀.

$$
f(z)=A \varphi\left(\begin{array}{c}
z \\
R_{p} \varepsilon
\end{array}\right)
$$

où $A \neq \mathrm{o},|\varepsilon|=\mathrm{I}$ et pour $\varrho^{(p)}=\mathrm{I}-\left(\frac{\mathrm{I}}{2}\right)^{\frac{1}{p}}$ :

$$
\varphi(z)=-2 \frac{\left(z-\rho^{(p)}\right)^{p}}{\mathbf{I}-z}
$$


C. Il existe une suite infinie de polynomes $f_{m}(z), m=\mathrm{I}, 2, \ldots$ pour lesquels les expressions dans les premiers membres des inégalités (I 7, 2), (17,3) convergent pour $m \rightarrow \infty$ resp. vers $\mathrm{I}-\left(\frac{\mathrm{I}}{2}\right)^{\frac{1}{p}}, \frac{\mathrm{I}}{2}$.

18. Avant de donner la démonstration du théorème VII, qui utilise la théorie de fonctions, nous allons démontrer le théorème suivant plus 》faible» mais dont la démonstration entre complètement dans le cadre de l'algèbre élémentaire:

VIII. Sous les conditions du théorème VII on a, si $f(\varepsilon)$ se réduit à un polynôme d'un degré $n \geqq p$ :

$$
\frac{\left|\zeta_{p}\right|}{R_{p}} \geqq\left(\frac{3}{2}\right)^{\frac{1}{p}}-\mathrm{I}
$$

$$
\prod_{x=1}^{p}\left(\mathrm{I}+\frac{\left|\zeta_{x}\right|}{R_{p}}\right) \geqq \frac{3}{2} .
$$

(I $8, \mathrm{I})$ est une conséquence immédiate de $(18,2)$, car, $\mathrm{I}+\frac{\left|\zeta_{p}\right|}{R_{p}}$ étant le facteur maximum des $p$ facteurs du produit de $(18,2)$, il résulte évidemment de $(18,2)$ que

$$
\left(\mathrm{I}+\frac{\left|\zeta_{p}\right|}{R_{p}}\right)^{p} \geqq \frac{3}{2}, \quad \mathrm{I}+\frac{\left|\zeta_{p}\right|}{R_{p}} \geqq\left(\frac{3}{2}\right)^{\frac{1}{p}} .
$$

Posons

$$
R_{p}=R
$$

et enfermons $p$ entre deux indices principaux consécutifs

alors on aura évidemment:

$$
n_{\mu-1}<p \leqq n_{\mu}
$$

$$
R=R_{n_{\mu}}, \quad n_{\mu-1}=p-\alpha, \quad \alpha \geqq \mathrm{I}
$$

et il résulte de $\left(15,5^{\circ}\right)$ que

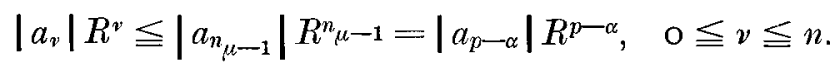

En divisant $f(z)$ par une constante on peut faire le produit de droite égal à un, de sorte que l'on peut supposer dès le début que les relations suivantes 
sont satisfaites.

Considérons maintenant le polynôme

$$
h(z)=\prod_{v=1}^{p}\left(z-\zeta_{v}\right)=\sum_{v=0}^{p} b_{v} R^{-v} z^{v}
$$

Il est majoré par $\prod_{v=1}^{p}\left(z+\left|\zeta_{v}\right|\right)$. On a donc pour $z=R=R_{p}$

$$
\sum_{\nu=0}^{p}\left|b_{\nu}\right| \leqq \prod_{\nu=1}^{p}\left(R+\left|\zeta_{\nu}\right|\right)=R^{p} \prod_{\nu=1}^{p}\left(\mathrm{I}+\frac{\left|\zeta_{\nu}\right|}{R}\right)
$$

et il suffit de démontrer que

$$
\sum_{v=0}^{p}\left|b_{\nu}\right| \geqq \frac{3}{2} R^{p}
$$

ou bien, puisque $b_{p}=R^{p}$, que

$$
\sum_{\nu=0}^{p-1}\left|b_{v}\right| \geqq \frac{R^{p}}{2}=\frac{b_{p}}{2}
$$

I9. Développons maintenant le quotient $\frac{f}{h}$ suivant les puissances de $z$ :

$$
\frac{f(z)}{h(z)}=\sum_{v=0}^{n-p} B_{\nu} R^{-v} z^{v}
$$

Désignons $\underset{v}{\operatorname{Max}}\left|B_{v}\right|$ par $B$ et soit $q$ un indice pour lequel $(19,1)$

$$
\operatorname{Max}_{v}\left|B_{\nu}\right|=B=\left|B_{q}\right|
$$

En comparant les coefficients des mêmes puissances de $z$ des deux côtés de la formule

exprimons $a_{p+q}$ et $a_{p-\alpha}$ :

$$
f(z)=h(z) \sum_{v=0}^{n-p} B_{\nu} R^{-v} z^{v}
$$

$$
a_{p+q}=\left(b_{p} B_{q}+b_{p-1} B_{q+1}+\cdots+b_{0} B_{p+q}\right) R^{-(p+q)}
$$

$$
a_{p-\alpha}=\left(b_{p-\alpha} B_{0}+b_{p-\alpha-1} B_{1}+\cdots+b_{0} B_{p-\alpha}\right) R^{-(p-\alpha)},
$$

où $B_{\mu}=$ o pour $\mu>n-p$. Il résulte de $(19,3)$ en vertu de $(\mathrm{r} 8,4)$ 


$$
\mathrm{I}=\left|a_{p-\alpha}\right| R^{p-\alpha} \leqq B \sum_{\nu=0}^{p-\alpha}\left|b_{v}\right| \leqq B \sum_{\nu=0}^{p-1}\left|b_{v}\right|
$$

$(19,4)$

$$
B \geqq \frac{\mathrm{I}}{\sum_{\nu=0}^{p-1}\left|b_{\nu}\right|}
$$

D'autre part en utilisant (I 8,4$)$ pour $\nu=p+q$ il résulte de (I9, 2) que

$$
\mathrm{I} \geqq\left|a_{p+q}\right| R^{p+q} \geqq\left|B_{q}\right|\left|b_{p}\right|-\left|b_{p-1} B_{q+1}+\cdots+b_{0} B_{p+q}\right|,
$$

donc par $(19,1)$ et $(19,4)$

$$
\begin{gathered}
\mathrm{I} \geqq B\left(b_{p}-\sum_{\nu=0}^{p-1}\left|b_{v}\right|\right), \\
\mathrm{I} \geqq \frac{b_{p}-\sum_{v=0}^{p-1}\left|b_{\nu}\right|}{\sum_{\nu=0}^{p-1}\left|b_{\nu}\right|},
\end{gathered}
$$

d'où $(18,5)$,

C. Q. F. D.

2o. Dêmonstration de VII. On peut supposer $R_{p}=\mathrm{I}$ pour l'indice $p$ en question, le cas général se ramenant à celui-ci par la transformation $z=R_{p} z^{\prime}$, dont l'effet est de diviser par $R_{p}$ toutes les inclinaisons numériques et les racines de $f(z)$. Alors le côté correspondant du diagramme de Newton $D$ de $f(z)$ est parallèle à l'axe des $x$ et en divisant $f(z)$ par une constante on amène ce côté sur l'axe des $x$. On peut donc supposer que le segment $\langle p-\mathrm{I}, p\rangle$ de l'axe des $x$ appartient à $D$.

Supposons que ce côté de $D$ commence en $p-\alpha(\alpha \geqq \mathrm{I})$. Alors on a $(20,1)$

$$
\begin{gathered}
\left|a_{\nu}\right| \leqq \mathrm{I}, \quad 0 \leqq \nu<\infty, \\
\left|a_{p-\alpha}\right|=\mathrm{I} .
\end{gathered}
$$$$
(20,2)
$$

Développons l'expression $(20,3)$

$$
F(z)=\frac{\mathrm{I}}{\prod_{x=1}^{p}\left(\mathrm{I}-\frac{\zeta_{x}}{z}\right)}
$$

suivant les puissances décroissantes de $z$ : 
$(20,4)$

$$
F(z)=\sum_{v=0}^{\infty} \frac{\sigma_{v}}{z^{\nu}}, \quad \sigma_{0}=\mathrm{I} .
$$

Ce développement est majoré par le développement de

$$
\frac{\mathrm{I}}{\prod_{x=1}^{p}\left(\mathrm{I}-\frac{\left|\zeta_{x}\right|}{z}\right)}=\sum_{\nu=0}^{\infty} \frac{t_{v}}{z^{v}}
$$

suivant les puissances décroissantes de $z$.

On peut évidemment supposer

$$
\left|\zeta_{p}\right|<\mathrm{I}
$$

puisque dans le cas contraire les inégalités à démontrer sont évidentes. Mais alors nos développements de $(20,3)$ et $(20,5)$ convergent pour $z=\mathrm{I}$ et l'on a

$$
\sum_{v=0}^{\infty}\left|\sigma_{v}\right| \leqq \frac{\mathrm{I}}{\prod_{x=1}^{p}\left(\mathrm{I}-\left|\zeta_{x}\right|\right)}
$$

Or, l'expression

$$
\frac{f(z) F^{\prime}(z)}{z^{p}}
$$

est évidemment holomorphe dans le cercle de convergence de $f(z)$. Donc la série de Laurent du produit $f(z) F(z)$ ne contient que les puissances de $z$ aux exposants $\geqq p$ :

$$
\sum_{\nu=0}^{\infty} a_{v} z^{\nu} \sum_{\mu=0}^{\infty} \frac{\sigma_{\mu}}{z^{\mu}}=z^{p} \sum_{\nu=0}^{\infty} b_{\nu} z^{\nu}
$$

En comparant ici le coefficient de $z^{p-\alpha}$ on a

$(20,7)$

$$
\sigma_{0} a_{p-\alpha}+\sigma_{1} a_{p-\alpha+1}+\cdots=0
$$

et, puisque $\sigma_{0}=\mathbf{I}$, d'après $(20,2)$

$$
\mathrm{I}=\left|a_{p-\alpha}\right| \leqq \sum_{\nu=1}^{\infty}\left|a_{p-\alpha+\nu} \sigma_{v}\right|
$$

donc, puisque d'après $(20, \mathrm{I})\left|a_{v}\right| \leqq \mathrm{I}$ :

18-39615. Acta mathematica. 72. Imprimé le 2 mars 1940. 
$(20,9)$

$$
\begin{gathered}
\mathrm{I} \leqq \sum_{v=1}^{\infty}\left|\sigma_{v}\right| \leqq \frac{\mathrm{I}}{\prod_{x=1}^{p}\left(\mathrm{I}-\left|\zeta_{x}\right|\right)}-\mathrm{I}, \\
\prod_{x=1}^{p}\left(\mathrm{I}-\left|\zeta_{x}\right|\right) \leqq \frac{\mathrm{I}}{2}, \quad\left(\mathrm{I}-\left|\zeta_{p}\right|\right)^{p} \leqq \frac{\mathrm{I}}{2}, \quad\left|\zeta_{p}\right| \geqq \mathrm{I}-\left(\frac{\mathrm{I}}{2}\right)^{\frac{1}{p}}
\end{gathered}
$$

e'est à dire les relations $(\mathrm{I} 7,2),(\mathrm{I} 7,3)$.

2 I. Dans quel cas le signe $=$ se présente-t-il dans les relations $($ I 7,2$)$ et $(\mathrm{I} 7,3)$ ? Soit $R_{p}=\mathrm{I}$. Supposons d'abord que l'on ait $\prod_{\nu=1}^{p}\left(\mathrm{I}-\left|\zeta_{v}\right|\right)=\frac{\mathrm{I}}{2}$. On aurait alors de $(20,9)$

$$
2=\sum_{\nu=0}^{\infty}\left|\sigma_{\nu}\right|=\frac{\mathrm{I}}{\prod_{x=1}^{p}\left(\mathrm{I}-\left|\zeta_{x}\right|\right)}=\sum_{v=0}^{\infty} t_{\nu}
$$

et puisque $\left|\sigma_{v}\right| \leqq t_{v}$ pour $\nu=0, \mathrm{I}, \ldots$.

$$
\begin{gathered}
\left|\sigma_{1}\right|=t_{1} \\
\left|\zeta_{1}+\cdots+\zeta_{p}\right|=\left|\zeta_{1}\right|+\cdots+\left|\zeta_{p}\right| .
\end{gathered}
$$

Donc on aurait en désignant par $\varepsilon$ un nombre de module I pour $\varrho_{1}>0, \ldots \varrho_{p}>0$ : $(2 \mathrm{I}, \mathrm{I}) \quad \zeta_{1}=\varepsilon \varrho_{1}, \zeta_{2}=\varepsilon \varrho_{2}, \ldots, \zeta_{p}=\varepsilon \varrho_{p} ; \sigma_{v}=\varepsilon^{v} t_{v} \quad(v=0, \mathrm{I}, \ldots)$

En divisant $f(z)$ par $a_{p-\alpha}$ on fait $a_{p-\alpha}=\mathrm{I}$; il résulte alors de $(20,7),(20,9)$

$$
\mathrm{I}=a_{p-\alpha}=-\sum_{v=1}^{\infty} a_{p-\alpha+v} \sigma_{\nu}=-\sum_{v=1}^{\infty} a_{p-\alpha+v} \varepsilon^{v} t_{\nu}, \quad \mathrm{I}=\sum_{v=1}^{\infty} t_{v}
$$

done

$$
a_{p-\alpha+v}=-\varepsilon^{-v}, \quad v=\mathrm{I}, 2, \ldots
$$

et le développement de $f(z)$ devient

$$
f(z)=\sum_{\nu=0}^{p-\alpha-1} a_{v} z^{\nu}+z^{p-\alpha}-z^{p-\alpha} \sum_{\nu=1}^{\infty}\left(\frac{z}{\varepsilon}\right)^{\nu}=\sum_{\nu=0}^{p-\alpha-1} a_{\nu} z^{\nu}+z^{p-\alpha}+\frac{z^{p-\alpha+1}}{z-\varepsilon}
$$

Or, $(z-\varepsilon) f(z)$ possède au moins $p$ racines. Donc, puisque c'est un polynôme dont le degré est au plus $p-\alpha+\mathrm{I}, \alpha \geqq \mathrm{I}$, on a $\alpha=\mathrm{I}$ et l'on obtient finale- 
ment, en remplaçant $z$ par $\varepsilon z$ et divisant tous les coefficients par $\varepsilon^{p-1}$, une expression de la forme

$(2 \mathrm{I}, 2)$

$$
\psi(z)=\sum_{\nu=0}^{p-2} a_{\nu} z^{v}+z^{p-1}+\frac{z^{p}}{z-\mathrm{I}}
$$

dont les $p$ racines positives $\varrho_{1}, \ldots, \varrho_{p}$ satisfont aux relations

$$
\prod_{x=1}^{p}\left(\mathrm{I}-\varrho_{x}\right)=\frac{\mathrm{I}}{2}, \quad 0<\varrho_{x}<\mathrm{I}, \quad x=\mathrm{I}, \ldots, p,
$$

et dont les $p-$ I premiers coefficients $a_{v}$ satisfont aux inégalités

$$
\left|a_{v}\right|<\mathrm{I}, \quad v=\mathrm{o}, \ldots, p-2 .
$$

Done on a

$$
\psi(z)=2 \frac{\prod_{x=1}^{p}\left(z-\varrho_{x}\right)}{z-\mathbf{I}}
$$

où les $\varrho_{x}$ satisfont à $(2 \mathrm{I}, 3)$.

22. Nous allons maintenant montrer que, chaque fois que les nombres positifs $\varrho_{x}$ satisfont à $(2 \mathrm{I}, 3)$, l'expression $(2 \mathrm{I}, 5)$ peut être mise sous la forme $(2 \mathrm{I}, 2)$, où $(2 \mathrm{I}, 4)$ est satisfaite.

En effet, soit

$(22, \mathrm{I}) \quad K(z)=\prod_{x=1}^{p}\left(z-\varrho_{x}\right)=z^{p}-b_{1} z^{p-1}+b_{2} z^{p-2}-\cdots+(-\mathrm{I})^{p} b_{p}$.

D'après $(2 \mathrm{I}, 3)$, la différence

$$
2 K(z)-z^{p}=z^{p}-2 b_{1} z^{p-1}+2 b_{2} z^{p-2}-\cdots
$$

s'annule pour $z=\mathrm{I}$, elle est donc divisible par $z-\mathrm{I}$. Le quotient $(2 \mathrm{I}, 5)$ est un polynôme de la forme

où les $a_{v}$ sont réels et

$$
z^{p-1}+\sum_{v=0}^{p-2} a_{v} z^{v}
$$

$$
a_{v}=\mathrm{I}-2 b_{1}+2 b_{2}-\cdots+(-\mathrm{I})^{p-\gamma-1} 2 b_{p-\gamma-1}
$$

$(22,2)$

$$
\frac{\mathrm{I}-a_{v}}{2}=b_{1}-b_{2}+\cdots+(-1)^{p-v} b_{p-v-1} .
$$


Il suffit donc de montrer que les sommes $(22,3)$

$$
b_{1}-b_{2}+\cdots-(-\mathrm{I})^{\mu} b_{\mu}, \quad \mu=\mathrm{I}, \ldots, p-\mathrm{I}
$$

sont comprises dans l'intervalle (O, I). Or, on a, d'après $(2 \mathrm{I}, 3)$

$$
2=\frac{\mathrm{I}}{\prod_{-x=1}^{p}\left(\mathrm{I}-\varrho_{x}\right)}>\mathrm{I}+\sum_{x=1}^{p} \varrho_{x}=\mathrm{I}+b_{1}, b_{1}<\mathrm{I}
$$

D'autre part, en appliquant au polynôme

$$
\prod_{x=1}^{p}\left(z+\varrho_{x}\right)=b_{0} z^{p}+b_{1} z^{p-1}+b_{2} z^{p-2}+\cdots, b_{0}=\mathbf{I}
$$

les inégalités de Newton, on a

$$
\begin{gathered}
b_{v}^{2} \geqq b_{v-1} b_{v+1}, \quad \frac{b_{v+1}}{b_{v}} \leqq \frac{b_{v}}{b_{v-1}} \\
\frac{b_{v+1}}{b_{v}} \leqq \frac{b_{1}}{b_{0}}=b_{1}<\mathrm{I}, \quad v=\mathrm{I}, \ldots, p-\mathrm{I}, \\
b_{1}>b_{2}>b_{3}>\cdots>b_{p}>0
\end{gathered}
$$

et les sommes $(22,3)$ sont d'après le théorème de Leibnitz comprises entre $b_{1}$ et $b_{1}-b_{2}$, donc entre o et $\mathrm{I}$, au sens étroit.

La relation

$$
\varrho_{p}=\mathrm{I}-\left(\frac{\mathrm{I}}{2}\right)^{\frac{1}{p}}
$$

n'est compatible avec $(2 \mathrm{I}, 3)$ que si $\varrho_{1}=\varrho_{2}=\cdots=\varrho_{p}$ et ceci est évidemment aussi suffisant. Donc la partie $B$. du théorème VII est démontrée.

Soit

$$
\varphi(z)=\sum_{v=0}^{p-2} a_{v} z^{v}+z^{p-1}-\sum_{v=0}^{\infty} z^{p+v}
$$

le développement de $(\mathrm{I} 7,9)$. Posons pour $m \geqq \mathbf{I}$

$$
f_{m}(z)=\sum_{\nu=0}^{p-1} a_{\nu} z^{\nu}+z^{p-1}-z^{p} \sum_{\nu=0}^{m} z^{\nu}
$$

Il résulte de $(2 \mathrm{I}, 4)$ que le diagramme de Newton de $f_{m}(z)$ contient le segment $\langle p-\mathrm{I}, m+p\rangle$ de l'axe des $x$. Donc on a, pour chacun de ces polynômes $f_{m}(z)$, 


$$
R_{p}=\mathrm{I}
$$

D'autre part, on a, en désignant par $\zeta_{x}^{(m)}$ la $x^{\text {ième }}$ des racines de $f_{m}(z)$, ces racines étant rangées dans l'ordre des modules croissants:

Donc, pour $m \rightarrow \infty$

$$
\zeta_{x}^{(m)} \rightarrow \varrho, \quad x=\mathrm{I}, \ldots, p
$$

$$
\frac{\zeta_{p}^{(m)}}{R_{p}} \rightarrow \varrho=\mathrm{I}-\left(\frac{\mathrm{I}}{2}\right)^{\frac{1}{p}}, \quad \prod_{x=1}^{p}\left(\mathrm{I}-\frac{\left|\zeta_{x}^{(m)}\right|}{R_{p}}\right) \rightarrow(\mathrm{I}-\varrho)^{p}=\frac{\mathrm{I}}{2}
$$

et la démonstration du théorème VII est achevée.

23. Ajoutons, pour finir ce $\S$, quelques remarques relatives aux rapports des théorèmes VII, VIII.

Tout d'abord il est facile de voir que l'inégalité (r8, I) est moins précise que $(\mathrm{I} 7,2) . \quad$ En effet, $p \varrho^{(p)}=p\left(\mathrm{I}-\left(\frac{\mathrm{I}}{2}\right)^{\frac{1}{p}}\right)$ croît avec $p \geqq \mathrm{I}$ et l'on a

$$
\frac{\mathrm{I}}{2} \leqq p \varrho^{(p)}<\lim _{p \rightarrow \infty} p \varrho^{(p)}=\lg 2
$$

Car, on a, en posant

$$
t(\alpha)=\frac{1-2^{-\alpha}}{\alpha}
$$

pour $\mathrm{o}<\alpha \leqq \mathrm{I}$ :

$$
\alpha^{2} 2^{\alpha} t^{\prime}(\alpha)=-\left(2^{\alpha}-\mathbf{I}-\alpha \lg 2\right)
$$

Or, l'expression entre parenthèses est le reste du développement de $e^{\alpha \text { lg } 2}$. Elle est donc positive. Donc, pour $0<\alpha<\mathrm{I}$,

$$
\begin{array}{r}
\frac{t^{\prime}(\alpha)<0,}{2}=t(\mathrm{I}) \leqq t(\alpha)<\lim _{\alpha \downarrow 0} t(\alpha)=\lg 2, \\
\frac{\mathrm{I}}{2} \leqq t\left(\frac{\mathrm{I}}{p}\right)=p \varrho<\lg 2 .
\end{array}
$$

D'autre part l'expression $p\left(\left(\frac{3}{2}\right)^{\frac{1}{p}}-\mathrm{I}\right)$ décroît avec $p$ croissant et

$$
\lg \frac{3}{2}=\lim _{p \rightarrow \infty} p\left(\left(\frac{3}{2}\right)^{\frac{1}{p}}-\mathrm{I}\right)<p\left(\left(\frac{3}{2}\right)^{\frac{1}{p}}-\mathrm{I}\right) \leqq \frac{\mathrm{I}}{2}
$$


Car, en posant pour $0<\alpha \leqq \mathrm{I}$

$$
s(\alpha)=\frac{\left(\frac{3}{2}\right)^{\alpha}-\mathrm{I}}{\alpha}=\frac{\mathrm{I}}{\alpha}\left(e^{q \alpha}-\mathrm{I}\right), \quad q=\lg \frac{3}{2},
$$

on a, en utilisant l'expression du reste du développement taylorien de $e^{-x}$

$$
e^{-q \alpha} \alpha^{2} s^{\prime}(\alpha)=e^{-q \alpha}-\mathrm{I}+q \alpha=\frac{q^{2} \alpha^{2}}{2} e^{-\theta q \alpha},
$$

pour un $\theta, \circ<\theta<\mathrm{I}$. Done $s^{\prime}(\alpha)$ est positif, d'où $(23,2)$. Done en particulier la borne de $(\mathrm{I} 7,2)$ est toujours $\geqq \frac{\mathrm{I}}{2 p}$, tandis que celle de $(\mathrm{I} 8, \mathrm{I})$ reste toujours $\leqq \frac{I}{2 p}$, les signes d'égalité étant exclus pour $p>\mathrm{I}$.

Quant aux relations $(\mathrm{I} 7,3)$ et $(\mathrm{I} 8,2)$, la seconde est une conséquence de la première. En effet posons pour simplifier l'écriture

$$
\frac{\left|\zeta_{x}\right|}{R_{p}}=\varrho_{x}, \quad S_{1}=\prod_{x=1}^{p}\left(\mathrm{I}-\varrho_{x}\right), \quad S_{2}=\prod_{x=1}^{p}\left(\mathrm{I}+\varrho_{x}\right) .
$$

On a évidemment

$$
S_{1}+S_{2}=2\left(\mathrm{I}+\Sigma \varrho_{1} \varrho_{2}+\Sigma \varrho_{1} \varrho_{2} \varrho_{3} \varrho_{4}+\cdots\right)
$$

où les sommes entre parenthèses sont les fonctions symétriques élémentaires des dimensions $2,4, \ldots$ Il en résulte pour $p>\mathrm{I}$, chaque $\varrho_{v}$ étant positif, que

$$
S_{1}+S_{2}>2, \quad S_{2}>2-S_{1}
$$

donc puisque d'après $(17,3), S_{1} \leqq \frac{1}{2}$ :

$$
S_{2}>\frac{3}{2}
$$

ce qui est la rélation $(18,2)$ où le signe $=$ est exclu. D'autre part on voit aisément que pour aucun $p>$ I la constante $\frac{3}{2}$ dans la relation $(18,2)$ ne peut être amélioré. En effet, en soumettant les $p$ nombres positifs $\varrho_{1}, \ldots, \varrho_{p}$ aux conditions $(2 \mathrm{I}, 3)$, on est assuré que $S_{1}=\frac{\mathrm{I}}{2}$. D'autre part, en faisant tendre $\varrho_{1}, \ldots, \varrho_{p-1}$ vers o et $\varrho_{p}$ vers I, l'expression de droite en $(23,3)$ tend vers 2 et $S_{2}$ vers $\frac{3}{2}$. 


\section{§ 6. Corollaires du théorème fondamental. Equations des degrés \& et 3.}

24. IX. On a sous les hypothèses du théorème VII, si $f(z)$ se réduit à un polynôme du degré exact $n$, pour les racines $\left(\mathrm{I} 7, \mathrm{I}^{\circ}\right)$ de $f(z)$ :

$(24,1)$

$$
\frac{\mathrm{I}}{2}<\frac{\left|\zeta_{1}\right|}{R_{1}} \leqq n
$$

$(24,2)$

$$
\frac{\mathrm{I}}{n} \leqq \frac{\left|\zeta_{n}\right|}{R_{n}}<2
$$

$$
\mathrm{I}-\left(\frac{\mathrm{I}}{2}\right)^{\frac{1}{p}}<\frac{\left|\zeta_{p}\right|}{R_{p}}<\frac{\mathrm{I}}{\mathrm{I}-\left(\frac{\mathrm{I}}{2}\right)^{n-p+1}}, p=2, \ldots, n-\mathrm{I}^{1}
$$

Démonstration: Si $R_{1}<R_{2}$, on a

donc $\frac{\left|\zeta_{1}\right|}{R_{1}} \leqq n$.

$$
\frac{\mathrm{I}}{R_{1}}=\left|\frac{a_{1}}{a_{0}}\right|=\left|\sum_{v=1}^{n} \frac{\mathrm{I}}{\zeta_{v}}\right| \leqq \frac{n}{\left|\zeta_{1}\right|}
$$

Si, plus généralement

\begin{tabular}{|c|c|c|}
\hline$p$ & $\varrho^{(p)}$ & $\frac{\mathrm{I}}{\rho^{(p)}}$ \\
\hline 2 & 0,292893 & $3,4 I_{4} 2 \mathrm{~T}$ \\
\hline 3 & 0,206299 & 4,84732 \\
\hline 4 & $0,159 x_{04}$ & $6,2852 \mathrm{x}$ \\
\hline 5 & 0,129449 & 7,72502 \\
\hline 6 & O, I09 IOI & 9,16579 \\
\hline 7 & 0,094276 & 10,607 II \\
\hline 8 & 0,082996 & I 2,04878 \\
\hline 9 & $0,074 \quad 125$ & I 3,49067 \\
\hline ro & 0,066967 & I 4,93273 \\
\hline
\end{tabular}

on a

$$
R_{1}=R_{2}=\cdots=R_{n_{1}}<R_{n_{1}}+\mathrm{I}
$$

1 Voici une table des valeurs de $\rho(p)=\mathrm{I}--\int^{p} \frac{\mathrm{I}}{2}$ et $\frac{\mathrm{I}}{\rho^{(p)}}$ pour $p=2, \ldots$, I 


$$
R_{1}^{-n_{1}}=\left|\frac{a_{n_{1}}}{a_{0}}\right|=\left|\sum \frac{\mathrm{I}}{\zeta_{1} \ldots \zeta_{n}}\right| \leqq\left(\begin{array}{l}
n \\
n_{1}
\end{array}\right)\left|\zeta_{1}\right|^{n_{1}} \leqq\left(\frac{n}{\left|\zeta_{1}\right|}\right)^{n_{1}}
$$

et $\frac{\left|\zeta_{1}\right|}{R_{1}} \leqq n . \quad$ L'inégalité $\frac{\left|\zeta_{1}\right|}{R_{1}}>\frac{1}{2}$ s'obtient pour $p=\mathrm{I}$ de $(\mathrm{I} 7,2)$, le signe $=$ n'étant jamais valable pour un polynôme.

On tire $(24,2)$ de $(24, \mathrm{I})$ en remplaçant $f(z)$ par $z^{n} f\left(\frac{\mathrm{I}}{z}\right)$. De même, les bornes supérieures de $(24,3)$ s'obtiennent des bornes inférieures données par le théorème VII en appliquant ce théorème à $z^{n} f\left(\frac{\mathrm{I}}{z}\right)$.

25. Remarques sur le théorème IX.

I) La borne supérieure de $(24,2)$ peut être remplacée par une borne supérieure exacte dépendant de $n$. On peut évidemment supposer $R_{n}=\mathrm{I}$. Alors on a dans $(17,1)$

$$
a_{n}=\mathrm{I}, \quad\left|a_{v}\right| \leqq \mathrm{I} \quad(0 \leqq \nu \leqq n-\mathrm{I})
$$

Donc, d'après un théorème de Cauchy on obtient une borne supérieure pour les racines de $f(z)$ par la seule racine positive $\mu_{n}$ de l'équation

$$
x^{n}-x^{n-1}-x^{n-2}-\cdots-x-\mathrm{I}=\mathrm{o} .
$$

Done, dans les hypothèses du théorème IX

$$
\frac{\left|\zeta_{n}\right|}{R_{n}} \leqq \mu_{n}, \quad \frac{\left|\zeta_{1}\right|}{R_{1}} \geqq \frac{\mathbf{I}}{\mu_{n}}
$$

On a

$$
\mu_{2}=\frac{\mathrm{I}+\sqrt{5}}{2}=\mathrm{I}, 6 \mathrm{1} 8034 \ldots, \quad \mu_{3}=\mathrm{I}, 839286755 \ldots
$$

et

$$
\mu_{2}<\mu_{3}<\mu_{1}<\cdots, \quad \mu_{n} \uparrow 2
$$

pour $n \rightarrow \infty$. En effet l'équation pour $\frac{\mathrm{I}}{\mu_{n}}$ est

$$
\mathrm{I}-y-y^{2}-\cdots-y^{n}=\mathrm{o}
$$

et il en résulte immédiatement que les nombres $\frac{\mathrm{I}}{\mu_{n}}$ décroissent avec $n$ croissant et convergent vers $\frac{\mathrm{I}}{2}$, la racine de l'équation limite $\mathrm{I}=\frac{y}{\mathrm{I}-y}$. 
2) Il résulte de la monotonie de $t(\alpha)=\frac{1-2^{-\alpha}}{\alpha}$ établie au No. 23 que pour $p \geqq 2$

$$
\frac{\lg 2}{p}>\mathrm{I}-\left(\frac{\mathrm{I}}{2}\right)^{\frac{1}{p}} \geqq \frac{2\left(\mathrm{I}-\sqrt{\mathrm{I}} \begin{array}{c}
- \\
2
\end{array}\right)}{p}=\frac{2-\sqrt{2}}{p}=\frac{0,5857864 \ldots .}{p} .
$$

Des évaluations précédentes il suit

Corollaire au théorème IX. On a sous les hypothèses de IX pour $p=2, \ldots, n-\mathbf{I}$

$(25,3) \quad 2(n-p+\mathrm{I})>\left(\mathrm{I}+\sqrt{\frac{\mathrm{I}}{2}}\right)(n-p+\mathrm{I})>\frac{\left|\zeta_{p}\right|}{R_{p}}>\frac{2-\sqrt{2}}{p}>\frac{\mathrm{I}}{2 p}$.

26. On a évidemment pour l'épaisseur relative de la couronne circulaire, dans laquelle varie la racine $\zeta_{p}$ si l'on varie arbitrairement les arguments des coefficients du polynôme $f(z)$ en laissant les modules de $a_{\nu}$ fixes, les bornes suivantes:

$2 n$ pour $p=\mathrm{I}, p=n ;\left(\frac{\mathrm{I}}{2-\sqrt{2}}\right)^{2} p(n-p+\mathrm{I})=2,9142 \mathrm{I} 36 \ldots p(n-p+\mathrm{I})$

Or, on a évidemment

$$
\text { pour } 2 \leqq p \leqq n-\mathrm{I} \text {. }
$$

$$
p(n-p+\mathrm{I}) \leqq \frac{(n+\mathrm{I})^{2}}{4}
$$

donc, puisque $\frac{\mathrm{I}}{4} \cdot 2,9 \mathrm{I} 4 \ldots(n+\mathrm{I})^{2}=0,7285 \ldots(n+\mathrm{I})^{2}>2 n$ :

$\mathrm{X}$. Il existe pour chaque entier positif n un nombre $c_{n}, \mathrm{I}<c_{n}<0,73(n+\mathrm{I})^{2}$, ne dépendant que de $n$ tel que si l'on varie arbitrairement les arguments des coefficients d'un polynôme quelconque du degré $n$ en laissant les modules des coefficients fixes, la $p^{i e m e}$ racine de ce polynôme, les racines étant rangées dans l'ordre des modules croissants, reste dans une couronne circulaire de centre à l'origine d'épaisseur relative $c_{n}<0,73(n+1)^{2}$.

Dans ce qui suit nous désignons par $c_{n}$ la borne inférieure de tous les $c_{n}$ du théorème $\mathbf{X}$.

Les bornes supérieures $d u$ théorème IX sont probablement trop grandes sauf la première. Celle-ci est évidemment atteinte pour le polynôme $\left(\mathrm{I}+\frac{z}{n}\right)^{n}$.

Quant au théorème $\mathbf{X}$, la borne $0,73(n+1)^{2}$ pour $c_{n}$ est probablement beaucoup trop élevée, mais on peut montrer aisément que le nombre $c_{n}$ pour $n \rightarrow \infty$ converge vers l'infini.

19-39615. Acta mathematica. 72. Imprimé lo 2 mars 1940. 
Considérons à cet effet le polynôme $s_{n}(z)=\left(\mathrm{I}+\frac{z}{n}\right)^{n}$ et le polynôme $s_{n}^{*}(z)$ qu'on obtient de $s_{n}(z)$ en y changeant les signes des puissances de $z$ aux exposants de la forme $4 v+1,4 v+2$. Avec $n \rightarrow \infty$ la suite des polynômes $s_{n}(z)$ converge uniformément vers $e^{z}$. La suite des polynômes $s_{n}^{*}(z)$ est majorée uniformément par $e^{z}$ et converge uniformément vers $\cos z-\sin z$. Donc, en désignant par $w_{n}$ une racine de module minimum de $s_{n}^{*}(z)$, on aura $\left|w_{n}\right| \rightarrow \frac{\pi}{4}$. Il en résulte que si l'on varie arbitrairement les arguments de tous les coefficients de $s_{n}(z)$ les racines de module minimum des polynômes ainsi obtenus sont situés dans une couronne circulaire de centre à l'origine dont l'épaisseur est $\geqq \frac{n}{\left|w_{n}\right|}$, ce qui pour $n \rightarrow \infty$ est équivalent à $\frac{4 n}{\pi}$, donc

$$
\underline{\lim } \frac{c_{n}}{n} \geqq \frac{4}{\pi}
$$

27. Il n'est pas dépourvu d'intérêt de calculer les bornes exactes correspondant aux théorèmes $I X$ et $X$ pour les cas des équations quadratiques et cubiques. Pour les équations du degré 2 les relations $(24,1),(24,2)$ et la remarque ajoutée au No. 25 sous I) permettent d'établir tout de suite des bormes exactes

$$
\frac{\sqrt{5}-1}{2} \leqq \frac{\left|\zeta_{1}\right|}{R_{1}} \leqq 2
$$

$$
\frac{1}{2} \leqq \frac{\left|\zeta_{2}\right|}{R_{2}} \leqq \frac{\sqrt{5}+\mathrm{I}}{2} .
$$

Si $R_{1}=R_{2}=R$, c'est-à-dire le diagramme de Newton se réduit à un segment de droite, on a

$$
\frac{\sqrt{5}-\mathrm{I}}{2} \leqq \frac{\left|\zeta_{1}\right|}{R} \leqq \mathrm{I} \leqq \frac{\left|\zeta_{2}\right|}{R} \leqq \frac{\sqrt{5}+\mathrm{I}}{2}
$$

En effet, dans ce cas on a $\left|\zeta_{1}\right|\left|\zeta_{2}\right|=R^{2}$ donc $\frac{\left|\zeta_{1}\right|}{R} \leqq \mathrm{I} \leqq \frac{\left|\zeta_{2}\right|}{R}$ et les bornes extrèmes $\frac{\sqrt{5} \pm \mathrm{I}}{2}$ sont atteintes pour l'équation $z^{2}-R z-R^{2}=0$.

La détermination de $c_{2}$ est aussi facile. On peut évidemment se borner aux équations de la forme 
$(27,4)$

$$
z^{2}-2 r e^{i \varphi} z+\mathrm{I}=0, \quad z+\frac{\mathrm{I}}{z}=2 r e^{i \varphi}, \quad r>0 .
$$

Alors on a $\left|\zeta_{1}\right| \leqq I \leqq\left|\zeta_{2}\right|$. Il suffit de considérer la couronne circulaire, dans laquelle varie $\zeta_{2}$ si l'on varie l'argument $\varphi$ arbitrairement, l'épaisseur relative de la couronne correspondante à $\zeta_{1}$ étant la même.

On a d'après Cauchy $\left|\zeta_{2}\right|^{2}-r\left|\zeta_{2}\right|-\mathrm{I} \leqq 0$, done

$$
\left|\zeta_{2}\right| \leqq r+\sqrt{r^{2}+\mathrm{I}}
$$

et cette borne est évidemment atteinte, pour chaque $r$, en posant $\zeta_{2}=\left(r+\sqrt{r^{2}+1}\right) i$, $\varphi=\frac{\pi}{2}$. Il s'agit maintenant de trouver une borne inférieure exacte pour $\left|\zeta_{2}\right|$. Si $r \leqq \mathrm{I},\left|\zeta_{\mathbf{g}}\right|$ peut devenir égal à un. En effet, l'équation

$$
z^{2}-2 r z+\mathrm{I}=\mathrm{o}
$$

a pour $0 \leqq r<$ I deux racines complexes de module I. Donc pour $r \leqq$ I l'épaisseur relative de la couronne circulaire en question est $r+\sqrt{r^{2}+I}$ et devient pour $r=\mathrm{I}$ égal ̀̀ $\mathrm{I}+\sqrt{2}$.

Soit maintenant $r>\mathrm{I}$. Alors pour l'équation $z^{2}-2 r z+\mathrm{I}=\mathrm{O}$ on a $\zeta_{2}=r+\sqrt{r^{2}-\mathrm{I}}$. Nous allons démontrer que pour $r>\mathrm{I}$ la borne inférieure de $\left|\zeta_{2}\right|$ est $r+\sqrt{r^{2}-\mathrm{I}}$, c'est-à-dire qu'aucun $z$ avec $\mathrm{I} \leqq|z|<r+\sqrt{r^{2}-\mathrm{I}}$ ne peut satisfaire à $(27,4)$.

En effet l'expression $\varrho+\sqrt{\varrho^{2}-\mathrm{I}}$ croît de I à $r+\sqrt{r^{2}-\mathrm{I}}$ si $\varrho$ croît de I à $r$. On peut donc mettre chaque $z$ avec $\mathrm{I} \leqq|z|<r+\sqrt{r^{2}-\mathrm{I}}$ sous la, forme

Alors on a

$$
z=\left(\varrho+\sqrt{\varrho^{2}-\mathrm{I}}\right) e^{i 9}, \quad \mathrm{I} \leqq \varrho<r .
$$

$$
\begin{gathered}
\frac{\mathrm{I}}{z}=\left(\varrho-\sqrt{\varrho^{2}-\mathrm{I}}\right) e^{-i \vartheta}, \\
\left|z+\frac{\mathrm{I}}{z}\right|=2 \sqrt{\varrho^{2}-\sin ^{2} \vartheta} \leqq 2 \varrho<2 r .
\end{gathered}
$$

Notre $z$ ne peut done pas satisfaire à l'équation $(27,4)$.

L'épaisseur relative de la couronne circulaire correspondant à notre $r>$ I est donc 
Done

$$
\frac{r+\sqrt{r^{2}+\mathrm{I}}}{r+\sqrt{r^{2}-\mathrm{I}}}=\frac{\mathrm{I}+\sqrt{\mathrm{I}+\frac{\mathrm{I}}{r^{2}}}}{\mathrm{I}+\sqrt{\mathrm{I}-\frac{\mathrm{I}}{r^{2}}}}<\mathrm{I}+\sqrt{2} .
$$

$$
c_{2}=\mathrm{I}+\sqrt{2}=2,4142 \mathrm{I} \ldots
$$

XI. Sous les hypothèses du théorème IX on a pour $n=2(27, \mathrm{I}),(27,2)$ et, si $R_{1}=R_{2}=R,(27,3)$. Enfin on a pour $c_{2}$ du théorème $\mathbf{X}(27,6)$. Toutes les bornes de ces inégalités sont exactes.

28. Passons maintenant aux équations cubiques:

XII. Sous les hypothèses du théorème IX on a pour $n=3$ :

$$
\frac{\mathrm{I}}{\mu_{3}} \leqq \frac{\left|\zeta_{1}\right|}{R_{1}} \leqq 3,
$$

$$
\mathrm{I} \leqq \frac{\left|\zeta_{2}\right|}{R_{2}} \leqq 3
$$

$$
\frac{\mathrm{I}}{3} \leqq \frac{\left|\zeta_{3}\right|}{R_{3}} \leqq \mu_{3}
$$

oì $\mu_{3}=\mathrm{r}, 839286755 \ldots$ est la racine positive de $z^{3}-z^{2}-z-\mathrm{I}=\mathrm{o}$. Toutes les bornes de ces inégalités sont exactes.

Démonstration. Les inégalitées $(28, \mathrm{I})$ et $(28,3)$ résultent immédiatement de $(24, I),(24,2)$ et des remarques faites au No. 25 sous I). Il reste à démontrer $(28,2)$. On peut évidemment supposer $R_{2}=$ I. Alors les bornes de $(28,2)$ sont atteintes pour les polynômes

$(28,4)$

$$
\left\{\begin{array}{l}
z^{3}+z^{2}-z+\frac{5}{27}=\left(z-\frac{1}{3}\right)^{2}\left(z+\frac{5}{3}\right) \\
-\frac{5}{27} z^{3}+z^{2}-z-1=\left(\frac{z}{3}-\mathrm{I}\right)^{2}\left(-\frac{5}{3} z-\mathrm{I}\right)
\end{array}\right.
$$

Par une transformation immédiate notre équation cubique peut être réduite à la forme

$$
t z^{3}+z^{2}-z+\varepsilon=0, \quad|t| \leqq \mathrm{I}, \quad|\varepsilon| \leqq \mathrm{I},
$$


Supposons que l'on ait

$(28,6)$

$$
\left|\zeta_{3}\right| \geqq\left|\zeta_{2}\right| \geqq 3,
$$

alors il résulte de notre équation

$$
-t \zeta_{3}=\mathrm{I}-\frac{\mathrm{I}}{\zeta_{3}}+\frac{\varepsilon}{\zeta_{3}^{2}}, \quad\left|t \zeta_{3}\right| \geqq \mathrm{I}-\frac{\mathrm{I}}{3}-\frac{\mathrm{I}}{9}=\frac{5}{9} .
$$

D'autre part on a évidemment

$$
-\frac{\mathrm{I}}{t}=\zeta_{1} \zeta_{2}+\zeta_{2} \zeta_{3}+\zeta_{3} \zeta_{1}
$$

ou, en remplaçant $\zeta_{1}$ par $-\frac{\varepsilon}{t \zeta_{2} \zeta_{3}}$,

$$
t \zeta_{2} \zeta_{3}=-\mathrm{I}+\varepsilon\left(\frac{\mathrm{I}}{\zeta_{2}}+\frac{\mathrm{I}}{\zeta_{3}}\right)
$$

Or, ici le module de l'expression à gauche est $\left|t \zeta_{3}\right|\left|\zeta_{2}\right| \geqq \frac{5}{9} \cdot 3=\frac{5}{3}$ et le module de l'expression de droite est $\leqq I+\frac{I}{3}+\frac{I}{3}=\frac{5}{3}$. Donc on a en divisant $(28,5)$ pour $z=\zeta_{a}$ par $\zeta_{3}^{2}$

$$
\left|\zeta_{2}\right|=3, \quad\left|t \zeta_{3}\right|=\frac{5}{9}, \quad\left|\mathrm{I}-\frac{\varepsilon}{\zeta_{2}}-\frac{\varepsilon}{\zeta_{3}}\right|=\frac{5}{3}, \quad\left|\mathrm{I}-\frac{\mathrm{I}}{\zeta_{3}}+\frac{\varepsilon}{\zeta_{3}^{2}}\right|=\frac{5}{9} .
$$

Or, de la troisième de ces relations il résulte évidemment

$$
\frac{\varepsilon}{\zeta_{3}}=\frac{\varepsilon}{\zeta_{2}}=-\frac{\mathrm{I}}{3}, \quad \zeta_{2}=\zeta_{3}, \quad|\varepsilon|=\mathrm{I}
$$

et alors de la quatrième relation

$$
\zeta_{2}=\zeta_{3}=3, \quad \varepsilon=-\mathrm{I},
$$

et $\operatorname{par}(28,7) t=-\frac{5}{27}$

Done on a en tout cas $\left|\zeta_{2}\right| \leqq 3$ et $\left|\zeta_{2}\right|=3$ seulement pour $\zeta_{2}=\zeta_{3}=3$, $\varepsilon=-\mathrm{I}, t=-\frac{5}{27}$, e'est-à-dire pour la deuxième des équations $(28,4)$. Donc on a dans $(28,2) \frac{\left|\zeta_{2}\right|}{R_{2}}=3$ seulement pour les polynômes qui s'obtiennent par une transformation de la forme $z=\alpha z^{\prime} d u$ second des polynômes $(28,4)$. 
La discussion de l'inégalité $\left|\zeta_{2}\right| \geqq \frac{\mathrm{I}}{3}$ est ramenée immédiatement au cas que nous venons de traiter, en remplaçant $z$ par $\frac{\mathrm{I}}{z}$.

\section{$\S 7$. Evaluations des produits des racines.}

29. On peut ajouter aux estimations du théorème VII pour les quotients $\frac{\left|\zeta_{p}\right|}{R_{p}}$ quelques évaluations assez précises pour les produits de ces quotients, c'està-dire pour les expressions

$$
\frac{\left|\zeta_{1} \ldots \zeta_{p}\right|}{R_{1} \ldots R_{p}}=\frac{T_{p}}{T_{0}}\left|\zeta_{1} \ldots \zeta_{p}\right|
$$

en employant les notations du No. Io. Tout d'abord on a pour le cas d'un polynôme:

XIII. On a sous les hypothèses du théorème IX pour les racines $\left(17, \mathrm{I}^{\circ}\right) d u$ polynôme $f(z)$ :

$$
\frac{\left|\zeta_{1} \ldots \zeta_{p}\right|}{R_{1} \ldots R_{p}} \leqq\left(\begin{array}{l}
n \\
p
\end{array}\right), \quad I \leqq p \leqq n
$$

oì $R_{v}$ sont les inclinaisons numériques du diagramme de Newton de $(\mathbf{1} 7, \mathbf{1})$.

La borne de $(29,2)$ est exacte, le signe d'égalité pouvant être réalisé pour chaque $n$ et pour chaque $p, \mathrm{I} \leqq p \leqq n$.

En effet, considérons le polynôme

$$
\left|a_{0}\right| \prod_{v=1}^{n}\left(I+\frac{z}{\left|\zeta_{v}\right|}\right)
$$

qui est normal d'après le théorème II. Donc ce polynôme est une majorante de la majorante newtonienne de $f(z)$ et l'on a

$$
\frac{\mathrm{I}}{R_{1} \ldots R_{p}} \leqq \sum \frac{\mathrm{I}}{\left|\zeta_{1} \ldots \zeta_{p}\right|} \leqq \frac{\left(\begin{array}{c}
n \\
p
\end{array}\right)}{\left|\zeta_{1} \ldots \zeta_{p}\right|}
$$

d'où $(29,2)$. Le signe d'égalité dans $(29,2)$ est réalisé en posant pour un nombre positif $r: \zeta_{1}=\zeta_{2}=\cdots=\zeta_{n}=r$, puisque alors on peut évidemment poser 


$$
a_{p}=(-\mathrm{I})^{p}\left(\begin{array}{l}
n \\
p
\end{array}\right) r^{-p}, \quad T_{p}=\left(\begin{array}{l}
n \\
p
\end{array}\right) r^{-p}, \quad T_{0}=\mathrm{I}
$$

3o. Quant à la détermination d'une borne inférieure de $(29$, I), un résultat classique d'Hadamard, équivalent à l'inégalité

$$
\frac{\left|\zeta_{1} \ldots \zeta_{p}\right|}{R_{1} \ldots R_{p}} \geqq \frac{\mathrm{I}}{p+\mathrm{I}}
$$

vient d'être précisé par M. Pólya qui démontre au lieu de (3O, I):

$$
\frac{R_{1} \ldots R_{p}}{\left|\zeta_{1} \ldots \zeta_{p}\right|} \leqq \sqrt{\frac{(p+\mathrm{I})^{p+1}}{p^{p}}}<\sqrt{e(p+\mathrm{I})}
$$

par une déduction extrèmement courte et élégante. Cette démonstration sera reproduite au No. 3 I d'après une communication écrite de M. Pólya. Mais d'abord nous allons donner pour le cas des polynômes une démonstration purement algébrique d'un résultat un peut plus faible que (3०, I), à savoir

$(30,3)$

$$
\frac{R_{1} \ldots R_{p}}{\left|\zeta_{1} \ldots \zeta_{p}\right|} \leqq 2 p+\mathrm{I}
$$

XIV. On a sous les hypothèses du théorème IX la relation $(3 \circ, 3)$ pour $p=1,2, \ldots, n$.

Démonstration. On peut supposer $a_{0}=T_{0}=\mathrm{I}$. Il résulte des inégalités (I 8, I), démontrables par des considérations purement algébriques l'inégalité

$$
\frac{\mathrm{I}}{T_{p}} \frac{\mathrm{I}}{\left|\zeta_{1} \ldots \zeta_{p}\right|}=\frac{R_{1} \ldots R_{p}}{\left|\zeta_{1} \ldots \zeta_{p}\right|} \leqq \prod_{v=1}^{p} \frac{\mathrm{I}}{\left(\frac{3}{2}\right)^{\frac{1}{v}}-\mathrm{I}}
$$

Donc les quotients de gauche en $(30,3)$ possèdent une borne universelle ne dépendant que de $p$. Soit $h_{p}$ la borne inférieure de toutes ces bornes c'est-à-dire le nombre le plus petit ne dépendant que de $p$ tel que l'on ait

$$
\frac{\mathbf{I}}{T_{p}\left|\zeta_{1} \ldots \zeta_{p}\right|}=\frac{R_{1} \ldots R_{p}}{\left|\zeta_{1} \ldots \zeta_{p}\right|} \leqq h_{p}
$$

Considérons maintenant l'équation du degré $n$ aux racines $\zeta_{1}^{2}, \zeta_{2}^{2}, \ldots, \zeta_{n}^{2}$ : 


$$
f_{1}(z) \equiv a_{0}^{\prime}+a_{1}^{\prime} z+\cdots+a_{n}^{\prime} z^{n}
$$

où $\pm a_{\nu}^{\prime}=a_{v}^{2}-2 a_{v-1} a_{v+1}+2 a_{v-2} a_{v+2}-\cdots$,

$(30,5)$

$$
\left|a_{\nu}^{\prime}\right| \leqq T_{v}^{2}+2 T_{v-1} T_{v+1}+2 T_{v-2} T_{v+2}+\cdots .
$$

Ici les $T_{v}$ sont des coefficients (IO, I) de la majorante newtonienne de $f(z)$ et les $T_{v}$ et $a_{v}$ aux indices <o et $>n$ sont égaux à o. Pour les coefficients $T_{v}^{\prime}$ de la majorante newtonienne de $f_{1}(z)$ on a

$$
T_{v}^{\prime} \leqq(2 v+\mathrm{I}) T_{v}^{2}
$$

En effet on a d'après $(15,7) T_{v-x} T_{v+x} \leqq T_{v}^{2}$, donc

$$
\left|a^{\prime}{ }_{v}\right| \leqq(2 v+\mathrm{I}) T_{v}^{2}
$$

Or, le polynôme $\sum_{\nu=0}^{n}(2 \nu+1) T_{\nu}^{2} z^{\nu}$ est normal, en vertu de

$$
\frac{(2 \nu+1)^{2} T_{\nu}^{4}}{(2 \nu-\mathrm{I}) T_{\nu-1}^{2}(2 \nu+3) T_{\nu+1}^{2}}=\frac{(2 \nu+\mathrm{I})^{2}}{(2 \nu+\mathrm{I})^{2}-4}\left(\frac{T_{v}^{2}}{T_{v-1} T_{\nu+1}}\right)^{2}>\mathrm{I}
$$

Donc ce polynôme est aussi une majorante de la majorante newtonienne de $f_{1}(z)$ et l'on a $(30,6)$.

En appliquant $(3 \circ, 4)$ à $f_{1}(z)$, il résulte

$$
\begin{aligned}
& \mid \frac{\mathrm{I}}{\left|\zeta_{1} \ldots \zeta_{1}\right|^{2}} \leqq h_{p} T_{p}^{\prime} \leqq(2 p+\mathrm{I}) h_{p} T_{p}^{\prime}, \\
& \mid \overline{\zeta_{1} \ldots \zeta_{p} \mid} \leqq \sqrt{(2 p+\mathrm{I}) h_{p}} T_{p},
\end{aligned}
$$

et l'on a, puisque $h_{p}$ est la constante la plus petite pour laquelle $(30,4)$ a lieu:

$$
\begin{array}{cc}
h_{p} \leqq \sqrt{(2 p+\mathrm{I}) h_{p}}, \quad h_{p}^{2} \leqq(2 p+\mathrm{I}) h_{p}, & \\
h_{p} \leqq(2 p+\mathrm{I}), & \text { C. Q. F. D. }
\end{array}
$$

31. Pour démontrer $(30,2)$, M. Pólya part de l'inégalité suivante, que Landau a déduite $1905^{1}$ de la formule de Jensen et redémontrée I9I $3^{2}$ par un autre raisonnement plus direct:

1 LaNDAU (I), pp. 5-9.

2 Landau (2), pp. I11-I13. 
$(3 \mathrm{I}, \mathrm{I})$

$$
\frac{r^{p}}{\left|\zeta_{1} \ldots \zeta_{p}\right|} \leqq \frac{1}{\left|a_{0}\right|} \sqrt{\sum_{v=0}^{\infty}\left|a_{v}\right|^{2} r^{2 v}}
$$

où $r>0$ n'est assujetti qu'à la condition d'être plus petit que le rayon de convergence de $f(z)$. On a en évaluant dans (3I, I) les $\left|a_{\nu}\right|$ par les coefficients de la majorante newtonienne de $f(z)$

$$
\begin{gathered}
\left|a_{\nu}\right| \leqq \frac{\left|a_{0}\right| \frac{T_{v}}{T_{0}}=\frac{\left|a_{0}\right|}{R_{1} \ldots R_{v}}}{} \\
\frac{r^{2 p}}{\left|\zeta_{1} \ldots \zeta_{p}\right|^{2}} \leqq \sum_{\nu=0}^{\infty}\left(\frac{r}{R_{1}} \cdots \frac{r}{R_{v}}\right)^{2}
\end{gathered}
$$

Posons ici pour un $p$ fixe $\geqq$ I

alors on a évidemment

$$
r=\theta R_{p}, \quad \theta=\sqrt{\frac{p}{p+\mathrm{I}}},
$$

$$
\begin{gathered}
\frac{R_{p}^{2 p}}{\left|\zeta_{1} \ldots \zeta_{p}\right|^{2}} \theta^{2 p} \leqq \sum_{v=0}^{\infty} \theta^{2 v}\left(\frac{R_{p}}{R_{1}} \cdots \frac{R_{p}}{R_{v}}\right)^{2} \\
\left(\frac{R_{1} \ldots R_{p}}{\left|\zeta_{1} \ldots \zeta_{p}\right|}\right)^{2} \leqq \sum_{\nu=0}^{p-1} \theta^{2(v-p)}\left(\frac{R_{v+1}}{R_{p}} \cdots \frac{R_{p-1}}{R_{p}}\right)^{2}+\mathrm{I}+\sum_{\nu=p+1}^{\infty} \theta^{2(v-p)}\left(\frac{R_{p}}{R_{p+1}} \ldots \frac{R_{p}}{R_{v}}\right)^{2} \leqq \\
\leqq \sum_{v=0}^{p-1} \theta^{2(\nu-p)}+\mathrm{I}+\sum_{v=p+1}^{\infty} \theta^{2(\nu-p)}=\frac{\mathrm{I}}{\theta^{2 p}\left(\mathrm{I}-\theta^{2}\right)}=\frac{(p+\mathrm{I})^{p+1}}{p^{p}}-
\end{gathered}
$$

donc

$\mathrm{XV}$. (Théorème d'Hadamard-Pólya). Soit $(\mathrm{I} 7, \mathrm{I})$ une série entière sans zéro à l'origine, $\left(\mathrm{I} 7, \mathrm{I}^{\circ}\right)$ ses racines et $R_{v}$ les inclinaisons numériques de son diagramme de Newton. Alors, si pour un entier $p \geqq$ I $f(z)$ possède au moins $p$ zéros dans son cercle de convergence, on a $(30,2)$.

Sous les hypothèses du théorème IX on peut déduire une évaluation qui est dans la moitié des cas plus précise que $(30,2)$. Considérons en effet le polynôme

$$
f^{*}(z) \equiv \prod_{v=1}^{n}\left(z-\frac{\mathrm{I}}{\zeta_{v}}\right)=\frac{z^{n}}{a_{0}} f\left(\frac{\mathrm{I}}{z}\right)
$$

en posant

$$
\mathfrak{M}_{f^{*}}(z)=\sum_{v=0}^{n} T_{v}^{*} z^{\nu}
$$

20-39615. Acta mathematica. 72. Imprimé le 3 mars 1940 . 
on a d'après ce que nous avons dit au No. I 3

$$
T_{n-p}^{*}=\frac{T_{p}}{T_{0}}
$$

Appliquons $(3 \circ, 2)$ au polynôme $f^{*}(z)$ en remplaçant les $T_{\nu}^{*}$ par leurs valeurs. Il vient

$$
\frac{T_{n}}{T_{n-p}}\left|\zeta_{n} \ldots \zeta_{n-p+1}\right| \leqq \sqrt{\frac{(p+\mathrm{I})^{p+1}}{p^{p}}}
$$

Remplaçons ici $p$ par $n-p$ et utilisons la relation

Il vient

$$
\left|\zeta_{1} \ldots \zeta_{p}\right|\left|\zeta_{p+1} \ldots \zeta_{n}\right|=\frac{T_{0}}{T_{n}}
$$

$(3 \mathrm{I}, 3)$

$$
\frac{T_{0}}{T_{p}\left|\zeta_{1} \ldots \zeta_{p}\right|}=\frac{R_{1} \ldots R_{p}}{\left|\zeta_{1} \ldots \zeta_{p}\right|} \leqq \sqrt{\frac{(n-p+\mathrm{I})^{n-p+1}}{(n-p)^{n-p}}}
$$

Posons $r_{v}=\left|\zeta_{v}\right|, v=\mathrm{I}, \ldots,, n$. On peut écrire $(30,2)$ et $(3 \mathrm{I}, 2)$ dans la forme

$$
\begin{gathered}
r_{1} \ldots r_{p} \geqq \frac{T_{\mathbf{0}}}{T_{p}} \sqrt{\frac{p^{p}}{(p+\mathrm{I})^{p+1}}}, \\
r_{n-p+1} \ldots r_{n} \leqq \frac{T_{n-p}}{T_{n}} \sqrt{\frac{(p+\mathrm{I})^{p+1}}{p^{p}}} .
\end{gathered}
$$

Donc en divisant:

$(3 \mathrm{I}, 4)$

$$
\frac{r_{1} \ldots r_{p}}{r_{n-p+1} \ldots r_{n}} \geq \frac{T_{0}}{T_{p}} \frac{T_{n}}{T_{n-p}} \frac{p^{p}}{(p+\mathrm{I})^{p+1}}
$$

Or, si $p \leqq \frac{n}{2}$, on a évidemment $\frac{r_{1} \ldots r_{p}}{r_{n-p+1} \ldots r_{n}} \leqq\left(\frac{r_{p}}{r_{p+1}}\right)^{p}$, donc

$$
\frac{r_{p+1}}{r_{p}} \leqq\left(\frac{T_{p} T_{n-p}}{T_{0} T_{n}}\right)^{\frac{1}{p}}\left(\mathrm{I}+\frac{\mathrm{I}}{p}\right) \sqrt[p]{p+\mathrm{I}}, \quad p \leqq \frac{n}{2}
$$

Si l'on a $p \geqq \frac{n}{2}$ il résulte $n-p \leqq \frac{n}{2}$. Appliquons alors $(3 \mathrm{r}, 5)$ au polynôme $f^{*}(z)$ en remplaçant $p$ par $n-p$. Les modules des racines de $f^{*}(z)$ rangés dans l'ordre croissant sont $\frac{\mathrm{I}}{r_{n}}, \frac{\mathbf{I}}{r_{n-1}}, \ldots, \frac{\mathbf{I}}{r_{1}}$ donc aux numéros $n-p, n-p+\mathbf{I}$ 
correspondent les modules $\frac{\mathrm{I}}{r_{p+1}}, \frac{\mathrm{I}}{r_{p}}$. De l'autre côté on a

$$
\frac{T_{n-p}^{*} T_{p}^{*}}{T_{0}^{*} T_{n}^{*}}=\frac{T_{p} T_{n-p}}{T_{0} T_{n}}
$$

Done il vient

$$
\text { (3 I, 6) } \quad \frac{r_{p+1}}{r_{p}} \leqq\left(\frac{T_{p} T_{n-p}}{T_{0} T_{n}}\right)^{\frac{1}{n-p}}\left(\mathrm{I}+\frac{\mathrm{I}}{n-p}\right) \sqrt[n-p]{\sqrt{n-p+\mathrm{I}}}, \quad p \geqq \frac{n}{2} .
$$

En rassemblant nos résultats on peut énoncer

Corollaire au théorème XV. Si sous les conditions du théorème $\mathrm{XV} f(z)$ est un polynôme $d u$ degré exact $n$ on a à côté de $(3 \mathrm{O}, 2)$ l'inégalité $(3 \mathrm{I}, 3)$ et pour les quotients $\frac{r_{p+1}}{r_{p}}$ l'inégalité $(3 \mathrm{I}, 5)$ ou $(3 \mathrm{I}, 6)$ suivant que $p \leqq \frac{n}{2}$ ou $p \geqq \frac{n}{2}$.

Une relation analogue à $(3 \mathrm{I}, 5)$ et $(3 \mathrm{I}, 6)$ peut aussi être obtenue de $(24,3)$ en écrivant cette relation pour $p+\mathrm{I}$ et $p$ et en divisant la première par la seconde en combinant les termes convenablement. On obtient alors

$(3 \mathrm{I}, 7) \quad \varrho(p+\mathrm{I}) \varrho(n-p+\mathrm{I}) D_{p} \leqq \frac{r_{p+1}}{r_{p}} \leqq \frac{D_{p}}{\varrho(n-p) \varrho(p)}, \quad\left(\varrho(\nu)=\mathrm{I}-\left(\frac{\mathrm{I}}{2}\right)^{1}\right)$.

Dans cette inégalité le facteur $D_{p}$ de la borne supérieure a la valeur

$$
\frac{T_{p}^{2}}{T_{p-1} T_{p+1}} \leqq\left(\frac{T_{p} T_{n-p}}{T_{0} T_{n}}\right)^{\operatorname{Max}}\left(\frac{1}{p}, \frac{1}{n-p}\right)
$$

comme on voit aisément en exprimant tous les $T_{\mu}$ par $T_{0}$ et $R_{y}$. Mais le facteur numérique $\frac{\mathrm{I}}{\varrho(n-p) \overline{\varrho(p)}}$ est essentiellement plus grand que le coefficient numérique correspondant dans $(31,5)$ et $(31,6)$. Quant à la borne inférieure pour $\frac{r_{p+1}}{r_{p}}$, dès qu'elle sera applicable on obtiendra un résultat beaucoup plus précis $\mathrm{du}$ théorème $\mathrm{XX}$ que nous allons démontrer au $\$$ Io.

(Cont.) 